\title{
Effect of latitudinally displaced gravity wave forcing in the lower stratosphere on the polar vortex stability
}

\author{
Nadja Samtleben $^{1}$, Christoph Jacobi ${ }^{1}$, Petr Pišoft ${ }^{3}$, Petr Š́acha ${ }^{2,3,4}$, and Aleš Kuchař ${ }^{3}$ \\ ${ }^{1}$ Institute for Meteorology, Universität Leipzig, Stephanstr. 3, 04103 Leipzig, Germany \\ ${ }^{2}$ Institute of Meteorology and Climatology, Universität für Bodenkultur Wien, Gregor-Mendel-Straße 33, \\ 1180 Vienna, Austria \\ ${ }^{3}$ Department of Atmospheric Physics, Faculty of Mathematics and Physics, Charles University, V Holesovickach 2, \\ 18000 Prague 8, Czech Republic \\ ${ }^{4}$ EPhysLab, Faculty of Sciences, Universidade de Vigo, Campus As Lagoas, s/n, 32004 Ourense, Spain
}

Correspondence: Nadja Samtleben (nadja.samtleben@uni-leipzig.de)

Received: 24 January 2019 - Discussion started: 30 January 2019

Revised: 27 May 2019 - Accepted: 7 June 2019 - Published: 3 July 2019

\begin{abstract}
In order to investigate the impact of a locally confined gravity wave (GW) hotspot, a sensitivity study based on simulations of the middle atmosphere circulation during northern winter was performed with a nonlinear, mechanistic, general circulation model. To this end, we selected a fixed longitude range in the East Asian region $\left(120-170^{\circ} \mathrm{E}\right)$ and a latitude range from 22.5 to $52.5^{\circ} \mathrm{N}$ between 18 and $30 \mathrm{~km}$ for the hotspot region, which was then shifted northward in steps of $5^{\circ}$. For the southernmost hotspots, we observe a decreased stationary planetary wave (SPW) with wave number 1 (SPW 1) activity in the upper stratosphere and lower mesosphere, i.e., fewer SPWs 1 are propagating upwards. These GW hotspots lead to a negative refractive index, inhibiting SPW propagation at midlatitudes. The decreased SPW 1 activity is connected to an increased zonal mean zonal wind at lower latitudes. This, in turn, decreases the meridional potential vorticity gradient $\left(q_{y}\right)$ from midlatitudes towards the polar region. A reversed $q_{y}$ indicates local baroclinic instability, which generates SPWs with wave number 1 in the polar region, where we observe a strong positive Eliassen-Palm (EP) divergence. As a result, the EP flux increases towards the polar stratosphere (corresponding to enhanced SPW 1 amplitudes), where the SPWs with wave number 1 break, and the zonal mean zonal wind decreases. Thus, the local GW forcing leads to a displacement of the polar vortex towards lower latitudes. The effect of the local baroclinic instability indicated by the reversed $q_{y}$ also produces SPWs with wave number 1 in the lower mesosphere. The effect on the dynam-
\end{abstract}

ics in the middle atmosphere due to GW hotspots that are located northward of $50^{\circ} \mathrm{N}$ is negligible, as the refractive index of the atmosphere is strongly negative in the polar region. Thus, any changes in the SPW activity due to the local GW forcing are quite ineffective.

\section{Introduction}

During winter, the dynamics of the middle atmosphere are mainly dominated by the polar vortex. The polar vortex develops due to the lack of incoming solar radiation and is modified by the impact of atmospheric waves with different spatial and temporal scales (Douville, 2009). The most important characteristic of atmospheric waves is their ability to transport and deposit energy and momentum. In particular, gravity waves (GWs), which mainly develop in the troposphere, distribute energy and momentum throughout the whole atmosphere; thus, GWs maintain the circulation and the thermal structure of the upper atmosphere (Fritts and Alexander, 2003). They also contribute to turbulence and mixing between all vertical layers. Their most important sources are orography (Smith, 1985; Nastrom and Fritts, 1992), convection (Tsuda et al., 1994), jet sources (Plougonven and Zhang, 2014) or spontaneous adjustment processes (Fritts and Alexander, 2003). Strongly depending on the phase speed $c$ and the background wind $u$, GWs are able to propagate into the middle atmosphere. Due to the ex- 
ponentially decreasing density of the atmosphere, the GW amplitude exponentially increases with height if the GWs propagate conservatively under background conditions that are constant with height. Usually, the GW spectrum is already saturated in the stratosphere, which means that GW amplitudes cannot grow anymore and, according to the linear theory, partly break. This effect becomes stronger as their phase speed $c$ gets closer to the background wind $u$. If $c$ is equal to $u$, the GW encounters its critical line and cannot propagate anymore (Lindzen, 1981). Thus, GWs propagating in the opposite direction of the background wind are usually observed in the middle atmosphere. Moreover, GWs that are faster than the background wind are able to propagate, but they are mostly filtered out by the strong polar-night jet when $c$ becomes equal to $u$ (at the latest). In the mesosphere GWs, which are propagating in the opposite direction of $u$, saturate and deposit their momentum. For this reason, the wind reverses in the upper mesosphere and lower thermosphere (MLT) (Lindzen, 1981; Holton, 1982). The transfer of energy and momentum by breaking GWs is also called "GW drag".

Owing to the variety of their sources, GWs have a large spatial and temporal variability. To capture the global distribution of GWs, the potential energy $\left(E_{\mathrm{pot}}\right)$, momentum flux (MF) or stability indicators (Pišoft et al., 2018) can be estimated using satellite data (Ern et al., 2004; Fröhlich et al., 2007; Hoffmann et al., 2013; Schmidt et al., 2016). These numerous observational studies highlight a number of different local GW hotspots, which are mainly generated by orography and convection. The most common GW hotspots are the orographically induced GW hotspots near the Alps (Hierro et al., 2018), the Andes (Llamedo et al., 2009; Alexander et al., 2010; Lilienthal et al., 2017), the Antarctic Peninsula (Moffat-Griffin et al., 2010), the Himalayas (Kumar et al., 2012), the Mongolian Plateau (White et al., 2018), the Rocky Mountains (Lilly et al., 1982) and in the Scandinavian region (Kirkwood et al., 2010). Typically, satellite observations show a characteristic structure of enhanced GW activity in the subtropical stratosphere that is caused by deep convection over Southeast Asia, America, Africa or the Maritime Continent in the respective summer season (Jiang et al., 2004; Wright and Gille, 2011; Ern and Preusse, 2012). Reliable estimates of GW drag from observations are generally difficult. Several methods have been established to derive the GW drag from satellite (e.g., Ern et al., 2014, 2016) or radar measurements (e.g., Reid and Vincent, 1987); however, the uncertainties of these estimates are quite large.

Model studies have indicated that GWs can already break in the lower stratosphere (LS) (e.g., Plougonven et al., 2008; Constantino et al., 2015), which leads to an additional transfer of momentum and energy in this region. In connection with high planetary wave (PW) activity, this greatly affects the stability of the polar vortex and can cause a sudden stratospheric warming (SSW) (Albers and Birner, 2014). This effect has also been observed in satellite measurements that have shown enhanced GW drag before SSWs (Ern et al., 2016). Thus, an additional GW forcing may lead to a preconditioning of the polar vortex.

This study focuses on the role of the zonal position of localized GW breaking areas and their effects on the middle atmosphere dynamics. It is motivated by the findings of Šácha et al. (2015), who focused on the East Asian and North Pacific (EA/NP) region near Japan, where they observed a GW hotspot that was active during equinoxes and winter solstices. The GWs are orographically and convectively generated due to the topography located directly at the coastline and the warm Kuroshio Current. Šácha et al. (2015) analyzed the local instabilities by calculating the Richardson number and by analyzing reanalysis data and found that the GWs break in this area. Based on these results, they simulated the observed Asian GW breaking hotspot with a general circulation model (GCM) and analyzed its effect on the middle atmosphere circulation (Šácha et al., 2016). According to previous publications, e.g., by Smith (2003), Lieberman et al. (2013) or Matthias and Ern (2018), Šácha et al. (2016) observed a forcing of additional stationary planetary waves (SPWs) due to a longitudinally variable GW drag. We pursue this idea by shifting the EA/NP hotspot meridionally while keeping its longitude range fixed to obtain information about its impact on the middle atmosphere at different latitudinal positions. Therefore, the EA/NP GW hotspot is our starting point, from which we displace the GW hotspot towards lower and higher latitudes in $5^{\circ}$ steps. In Sect. 2 of this paper, we provide a brief description of the GCM and detail the implementation of the GW hotspot within the GCM. In Sect. 3 we describe and discuss the observed effects of the GW hotspots on the circulation of the middle atmosphere by analyzing the SPW activity and the propagation conditions. Finally, the conclusions and outlook are presented in Sect. 4.

\section{Numerical model experiments}

\subsection{Model description and setup}

To investigate the effect of localized GW breaking hotspots in the LS, simulations were performed using the Middle and Upper Atmosphere Model (MUAM, Pogoreltsev et al., 2007). MUAM is a nonlinear mechanistic 3-D grid point model, which is an updated version of the COMMA-LIM general circulation model (Fröhlich et al., 2003a, 2007; Jacobi et al., 2006). The model extends in 56 layers up to an altitude of about $160 \mathrm{~km}$ in logarithmic pressure height $z=-H \ln \left(p / p_{0}\right)$ with a constant scale height of $H=7 \mathrm{~km}$ and a reference pressure of $p_{0}=1000 \mathrm{hPa}$. Depending on the temperature profile, the logarithmic pressure height used can differ from the geometric height. However, at altitudes below $80 \mathrm{~km}$, this difference is negligibly small. At $110 \mathrm{~km}$ the deviation increases up to $5 \mathrm{~km}$, whereas the highest logarithmic pressure level of about $160 \mathrm{~km}$ may correspond to 
(a)

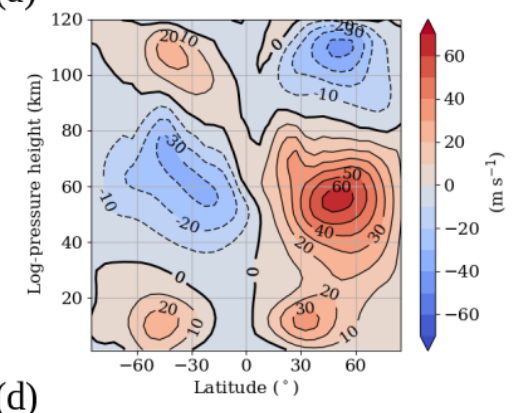

(d)

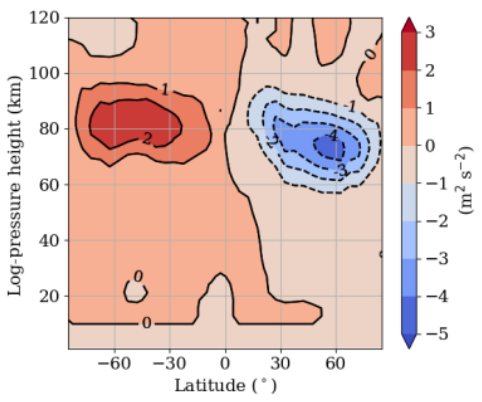

(b)

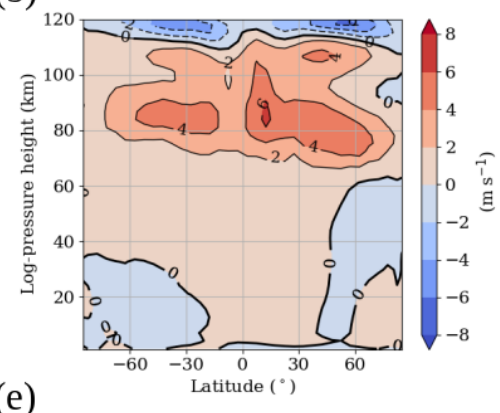

(e)

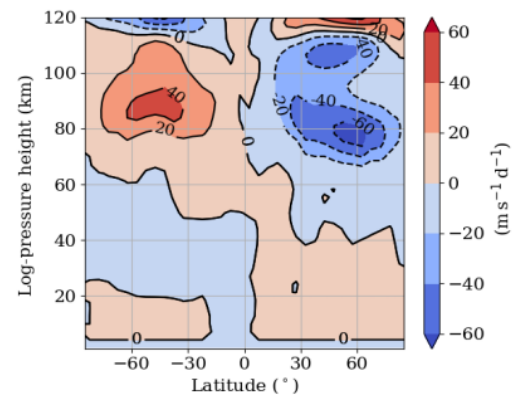

(c)

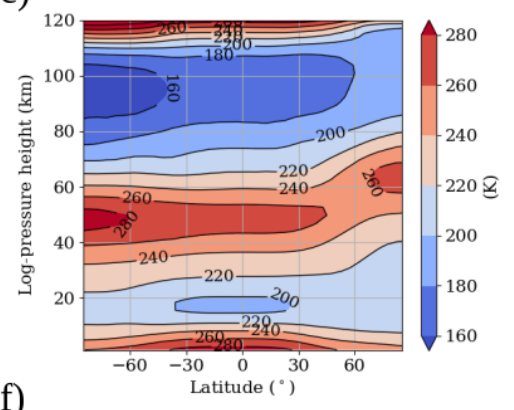

(f)

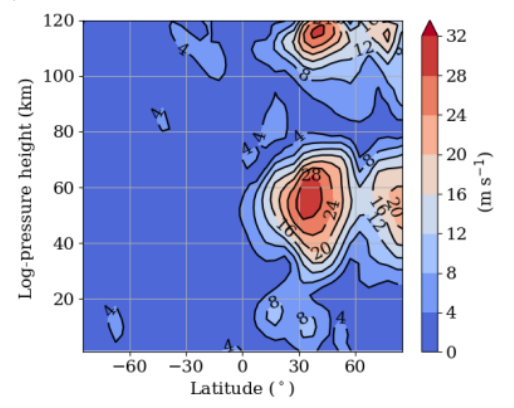

Figure 1. January zonal and monthly mean of the (a) zonal wind $\left(\mathrm{m} \mathrm{s}^{-1}\right)$, (b) meridional wind $\left(\mathrm{m} \mathrm{s}^{-1}\right)$, (c) temperature (K), (d) zonal GW fluxes $\left(\mathrm{m}^{2} \mathrm{~s}^{-2}\right)$, (e) zonal wind acceleration due to breaking GWs $\left(\mathrm{m} \mathrm{s}^{-1} \mathrm{~d}^{-1}\right)$ and (f) SPW 1 amplitude $\left(\mathrm{m} \mathrm{s}^{-1}\right)$ extracted from the zonal wind of the reference simulation.

a geometrical height between 300 and $400 \mathrm{~km}$. In the lowermost $10 \mathrm{~km}$, zonal mean temperatures are nudged to the 2000-2010 mean monthly mean ERA-Interim (Dee et al., 2011) zonal mean temperatures to correct the climatology of the troposphere, which is not included in the model in detail (Jacobi et al., 2015; Lilienthal et al., 2018). Furthermore, at $1000 \mathrm{hPa}$, which defines the lower boundary of the model, SPWs with wave numbers 1,2 and 3 are forced, which are extracted from the 2000-2010 mean ERA-Interim monthly temperature and geopotential reanalysis data. The horizontal resolution of the model is $5^{\circ}$ in latitude and $5.625^{\circ}$ in longitude and the vertical resolution is $2.842 \mathrm{~km}$. The model solves the primitive equations in flux form (e.g., Jakobs et al., 1986). MUAM includes parameterizations to simulate subgrid processes such as GWs, absorption of solar radiation or infrared cooling. The absorption of radiation is realized according to Strobel (1986). This parameterization is focused on the absorption processes due to trace gases such as $\mathrm{H}_{2} \mathrm{O}$ (absorber in the troposphere) as well as $\mathrm{CO}_{2}$ and $\mathrm{O}_{3}$ (absorbers in the stratosphere). Water vapor and ozone fields are prescribed. The heating rates are calculated by absorption bands representing the wavelength interval at which these trace gases absorb the atmospheric radiation. The infrared emission of $\mathrm{CO}_{2}$ is parameterized following Fomichev et al. (1998), and ozone infrared cooling in the $9.6 \mu \mathrm{m}$ band is calculated following Fomichev and Shved (1985).

GWs are parameterized after an updated linear scheme (Lindzen, 1981; Jakobs et al., 1986) with multiple breaking levels (Fröhlich et al., 2003b; Jacobi et al., 2006). GW amplitudes are included at an altitude of $10 \mathrm{~km}$ as a zonal mean with a global average of $1 \mathrm{~cm} \mathrm{~s}^{-1}$ for the vertical velocity perturbation. This value is weighted by a prescribed zonal mean GW amplitude distribution based on $E_{\text {pot }}$ data obtained from GPS radio occultation measurements (Šácha et al., 2015; Lilienthal et al., 2017). Although the $E_{\text {pot }}$ data still contain Kelvin waves and other possible wave structures with short vertical wavelengths, which may introduce biases, the GW amplitude distribution is more realistic than the hyperbolic tangent function of the latitude, which was used in earlier experiments (Jacobi et al., 2006), and leads to an improvement of the zonal mean GW climatology. It shows maximum GW amplitudes (not shown here) at the Equator (convectively generated GWs) and at midlatitudes (orographically induced GWs). At each grid point 48 waves are induced that propagate in eight different directions with six different phase speeds ranging from 5 to $30 \mathrm{~m} \mathrm{~s}^{-1}$.

In this configuration, based on January decadal mean (2000-2010) ERA-Interim reanalysis data, we create a reference simulation with a spin-up period of $270 \mathrm{~d}$ (days), in which the mean circulation is built up and different waves such as PWs and tides are generated. The declination and the ozone and carbon dioxide concentration are fixed to avoid further non-zonal structures being induced in addition to the enhanced GW forcing. The declination corresponds to 15 January (referring to the middle of the month), and the ozone and carbon dioxide data are taken from the year 2005 (referring to the middle of the decade). For the analysis, a time interval of $120 \mathrm{~d}$ with a temporal resolution of $2 \mathrm{~h}$ after 

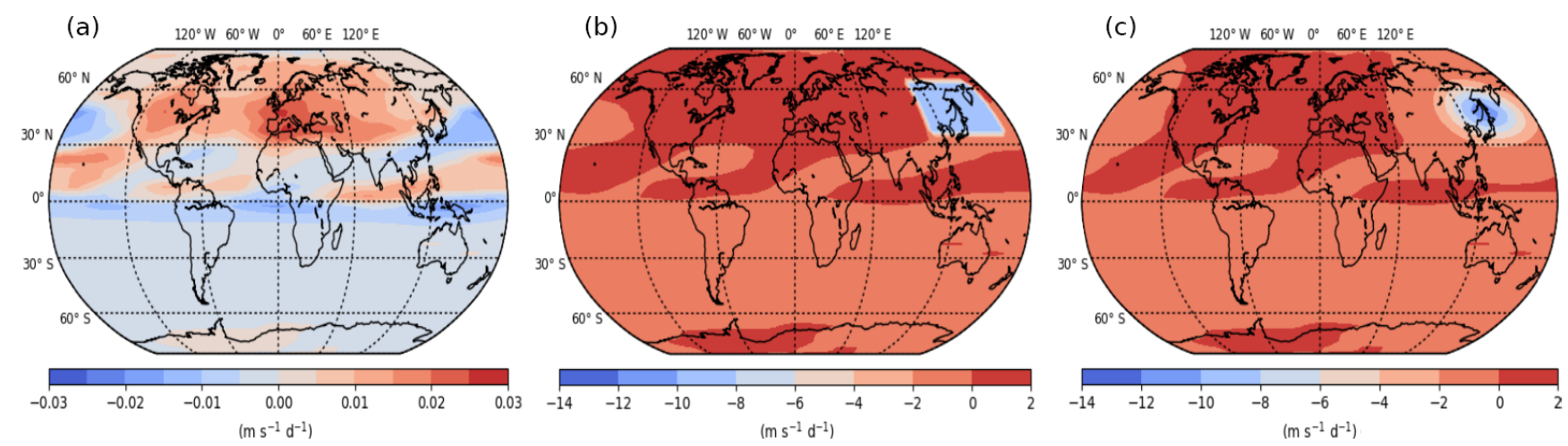

Figure 2. Zonal GW drag $\left(\mathrm{m} \mathrm{s}^{-1} \mathrm{~d}^{-1}\right)$ at $26.9 \mathrm{~km}$ for the reference (a) and the $\mathrm{H} 3$ hotspot simulation as a box (b) and as a Gaussian distribution (c) for the last $30 \mathrm{~d}$ of analysis. Note the different scale for panel (a).

the spin-up period was modeled. Šácha et al. (2016) have already analyzed the effect of the Asian hotspot with MUAM by performing a sensitivity study with regard to the strength of the GW forcing in the stratosphere. Their analysis time period was much shorter and the declination of the sun was different. They also nudged the model zonal mean temperature up to $30 \mathrm{~km}$. In this regard, our experimental setup might be considered superior to their simulations, especially as the nudging does not interfere with the GW forcing implemented in this new configuration. We refer to this reference simulation as "Ref".

The state of the middle atmosphere in the Ref simulation can be seen in Fig. 1, which shows the January zonal mean zonal (Fig. 1a) and meridional wind (Fig. 1b), the temperature (Fig. 1c), zonal GW flux (Fig. 1d), the zonal wind acceleration due to breaking GWs (Fig. 1e), and the SPW 1 amplitude extracted from the zonal wind (Fig. 1f) as latitude-height plots. Each parameter is presented up to an altitude of $120 \mathrm{~km}$ for the winter and summer hemisphere. The zonal wind in Fig. 1a generally reproduces reference climatologies like CIRA-86 (Fleming et al., 1988) or URAP (Swinbank and Ortland, 2003), but the winter mesospheric jet is overestimated by about $10-20 \mathrm{~m} \mathrm{~s}^{-1}$. The meridional circulation (Fig. 1b) extending from the summer to the winter mesopause has a maximum of $6 \mathrm{~m} \mathrm{~s}^{-1}$ at about $80 \mathrm{~km}$, which reproduces predictions by climatologies well (Portnyagin et al., 2004; Jacobi et al., 2009). Temperature (Fig. 1c) generally reproduces climatology values. The GW fluxes (Fig. 1d) maximize at about $80 \mathrm{~km}$, with a maximum of slightly above $-4 \mathrm{~m}^{2} \mathrm{~s}^{-2}$ in the Northern Hemisphere (NH) and $2 \mathrm{~m}^{2} \mathrm{~s}^{-2}$ in the Southern Hemisphere (SH). The corresponding zonal $\mathrm{GW}$ drag maximizes at the same altitude with about $-60 \mathrm{~m} \mathrm{~s}^{-1} \mathrm{~d}^{-1}\left(40 \mathrm{~m} \mathrm{~s}^{-1} \mathrm{~d}^{-1}\right)$ in the NH (SH) and is directed westward (eastward). The SPW 1 amplitude (Fig. 1f) extracted from the zonal wind shows maximum values at the border of the mesospheric jet maximum north of $30^{\circ} \mathrm{N}$ between 50 and $60 \mathrm{~km}$ and in the polar region. This fits quite well with observations, but the amplitudes are slightly underestimated due to the overestimated mesospheric jet, filtering some of the SPWs (Xiao et al., 2009).

\subsection{Experiment description}

Firstly, we reproduced the experiment of Šácha et al. (2016) to check if we still obtained similar results with the slightly modified setup. To represent the Asian GW breaking hotspot in the model, we enhanced the GW drag after model day 270 , i.e., after the spin-up, and ran the model for $120 \mathrm{~d}$ as in the Ref simulation. Hence, the zonal $\left(\mathrm{GWD}_{u}\right)$ and meridional $\left(G_{v}\right)$ GW drag and the heating due to breaking GWs $\left(G W D_{T}\right)$ were modified in the specific region of the observed GW breaking hotspot. In principle, the response to the GW drag would in turn alter the GW propagation and breaking conditions and, thus, the GW drag and its distribution. To avoid those feedback mechanisms, the GW parameterization scheme is turned off during the experiments, and the model is fed with the GW drag field from the Ref simulation. However, in the GW hotspot region, the GW drag is modified (as shown in Table 1). We intend to only analyze the steady-state impact of the local GW forcing that is not influenced by nonlinear effects.

As in Šácha et al. (2016), we located the GW breaking hotspot between 37.5 and $62.5^{\circ} \mathrm{N}$ and between 118.1 and $174.3^{\circ} \mathrm{E}$ in an altitude range between 18 and $30 \mathrm{~km}$. Note that the geographic positions refer to the model grid points; thus, at a latitudinal $5^{\circ}$ grid, the meridional size of the modeled hotspot is $30^{\circ}$. To avoid a total breakdown of the polar vortex and a fundamental change in middle atmosphere dynamics, which was already forced in the study by Šácha et al. (2016), we chose the more moderate case of $-10 \mathrm{~m} \mathrm{~s}^{-1} \mathrm{~d}^{-1}$ for $\mathrm{GWD}_{u},-0.1 \mathrm{~m} \mathrm{~s}^{-1} \mathrm{~d}^{-1}$ for $\mathrm{GWD}_{v}$ and a warming of $0.05 \mathrm{~K} \mathrm{~d}^{-1}$ for $\mathrm{GWD}_{T}$. We refer to this simulation as the $\mathrm{H} 3$ simulation, as will be described later. The distribution of the $\mathrm{GWD}_{u}$ of the Ref and the H3 simulations can be seen in Fig. 2a and b at an altitude of about $27 \mathrm{~km}$ for the last $30 \mathrm{~d}$ of analysis. We mainly concentrate on the last $30 \mathrm{~d}$ of analysis, as we focus on quasi-steady states and are 
not interested in short-term variabilities. The $\mathrm{GWD}_{u}$ of the Ref simulation varies between -0.025 and $+0.02 \mathrm{~m} \mathrm{~s}^{-1} \mathrm{~d}^{-1}$ in the GW hotspot region $\left(27.5-87.5^{\circ} \mathrm{N}, 118.1-174.3^{\circ} \mathrm{E}\right.$, $18-30 \mathrm{~km})$. Thus, the maximum value of the $\mathrm{H} 3$ simulation $\left(\mathrm{GWD}_{u}=-10 \mathrm{~m} \mathrm{~s}^{-1} \mathrm{~d}^{-1}\right.$ in the hotspot) is 500 times larger than the maximum westward (negative) value of the Ref simulation. The $\mathrm{H} 3$ mean value (mean $\mathrm{GWD}_{u}:-10 \mathrm{~m} \mathrm{~s}^{-1} \mathrm{~d}^{-1}$ ) is roughly 3300 times larger than that of the Ref simulation (mean $\mathrm{GWD}_{u}: 0.003 \mathrm{~m} \mathrm{~s}^{-1} \mathrm{~d}^{-1}$ ) within the region of the EA/NP hotspot. These maximum values of the $\mathrm{GWD}_{u}$ as well as those of the $\mathrm{GWD}_{v}$ and the $\mathrm{GWD}_{T}$ are summarized in Table 1 for the Ref and the GW hotspot simulations. In spite of the huge difference compared with the Ref simulation, the zonal GW forcing is moderate in terms of what is estimated from observations $\left(40 \mathrm{~m} \mathrm{~s}^{-1} \mathrm{~d}^{-1}\right.$ and more) and from GW parameterizations in this region (Šácha et al., 2018). Concerning the meridional GW drag and the heating due to breaking GWs, the maximum (mean) value of the $\mathrm{H} 3$ simulation is only 5 (100) times larger than that of the Ref simulation (not shown here). To investigate possible effects with regard to the position of the GW hotspot, we performed a sensitivity study. For this, we kept the longitude (118.1$\left.174.3^{\circ} \mathrm{E}\right)$ and altitude $(18-30 \mathrm{~km})$ range as well as the zonal extent of $25^{\circ}$ fixed, but varied the observed GW hotspot in $5^{\circ}$ steps from $27.5-52.5^{\circ} \mathrm{N}$ (simulation $\mathrm{H} 1$ ) to $62.5-87.5^{\circ} \mathrm{N}$ (simulation H8), while labeling the experiments in between as $\mathrm{H} 2$ through $\mathrm{H} 7$ (see Table 1).

To analyze the possible effects of the sharp transition zone between the unchanged and enhanced GW drag, additional simulations with a smoothed GW forcing were performed, using a 3-D Gaussian function with standard deviations of $10^{\circ}, 22.5^{\circ}$ and $5.684 \mathrm{~km}$ in the zonal, meridional and vertical directions, respectively. To get the same integral forcing as in the $\mathrm{H} 1-\mathrm{H} 8$ simulations, the size or the intensity of the local GW forcing as a Gaussian distribution needed to be adjusted. For our experiments, we mainly increased the strength of the local GW forcing and only slightly increased the size. The maximum values for the $\mathrm{GWD}_{u}, \mathrm{GWD}_{v}$ and $\mathrm{GWD}_{T}$ forcing as a 3-D Gaussian distribution were chosen to be $-13 \mathrm{~m} \mathrm{~s}^{-1} \mathrm{~d}^{-1},-0.13 \mathrm{~m} \mathrm{~s}^{-1} \mathrm{~d}^{-1}$ and $0.065 \mathrm{~K} \mathrm{~d}^{-1}$ (see Table 1), respectively. The 3-D Gaussian distribution for the H3 GW hotspot can be seen in Fig. 2c. In this paper we mainly concentrate on the 3-D GW hotspots shaped like a box when we analyze the effects on the middle atmosphere dynamics. For comparison, regarding the shape of the artificial GW forcing, we just focus on the $\mathrm{H} 3 \mathrm{GW}$ hotspot with Gaussian smoothed boundaries when we discuss the SPW modulation in Sect. 3.2, which may be affected by the GW hotspots with sharp boundaries.

When comparing the size of the GW hotspots it is obvious (can be seen in Fig. 5) that the area of the enhanced GW drag, which scales with the cosine of the latitude, decreases with increasing latitude. However, scaling the GW drag with latitude would lead to a much larger zonal mean GW drag at high latitudes and would result in changes in the circula- tion. Furthermore, the horizontal winds, which are affected by resulting nonlinear interactions, are scaled in the model equations. In the current approach, we conserve the ratio of enhanced and unchanged GW drag values within the respective latitudinal belt, which is more meaningful. Also, the horizontal wavelength of PWs becomes smaller with decreasing distance from the pole, meaning that the ratio of the width of the GW forcing and the horizontal wavelength of the PWs remains the same for the respective latitudinal belt. In the following, we will show that the spatial shape as well as the spatial size of the local GW forcing is not the most decisive factor when we compare the 3-D Gaussian distribution with the 3-D GW forcing shaped as a box. Thus, GW hotspots that are the same size may lead to comparable results.

\section{Results}

\subsection{Hotspot effect on the background circulation}

Figure $3 \mathrm{a}-\mathrm{h}$ show the zonal mean zonal wind difference between each GW hotspot simulation H1-H8 and the Ref simulation (in color) as well as the zonal mean zonal wind of the Ref simulation (as contour lines) in a latitude-height plot. The position of each GW hotspot is illustrated by a red box. All experiments (H1-H8) show negative zonal wind differences with a maximum wind decrease of more than $-10 \mathrm{~m} \mathrm{~s}^{-1}$ in the polar region. Positive differences can be observed equatorward from an imaginary line connecting the subtropical and polar-night jet centers, with a maximum difference of 8 to $10 \mathrm{~m} \mathrm{~s}^{-1}$. These zonal wind anomalies are consistent with a polar vortex that is shifted towards lower latitudes, and the wind reversal in the mesosphere is shifted upwards at lower latitudes. The strongest decrease in the zonal mean zonal wind in the polar region can be observed in the $\mathrm{H} 1$ simulation (Fig. 3a) and the strongest increase in zonal mean zonal wind at lower latitudes can be observed in the $\mathrm{H} 3$ simulation (Fig. 3c), the latter corresponds to the observed Asian GW hotspot. For GW hotspots with a southern edge north of $50^{\circ} \mathrm{N}$, the polar vortex is only slightly displaced towards lower latitudes. Thus, the effect of GW hotspots at higher latitudes is not as strong.

Figure 4 is arranged in the same manner as Fig. 3, but shows the temperature difference (in color) and the vertical wind difference (as contour lines). As expected, the temperature effect scales (both zonal and vertical) with the wind differences; thus, the H1-H3 simulations in Fig. 4a-c show the strongest temperature anomalies, and these once again decrease in magnitude for northward-shifted GW hotspots. Between 60 and $90^{\circ} \mathrm{N}$, the GW hotspot leads to a temperature increase at altitudes up to $30-35 \mathrm{~km}$, but to a decrease above. The zonal mean vertical wind difference shows generally negative anomalies between 15 and $30 \mathrm{~km}$ at higher latitudes, which indicates a stronger downward movement connected with an adiabatic warming in the lower part of the 
Table 1. Overview of the mean and maximum values of the zonal and meridional GW drag and heating by GWs for the reference and hotspot simulations as a 3-D box (H1-H8) and as a Gaussian distribution (Gauss). The mean and maximum values refer to the region (118.1-174.3 $3^{\circ} \mathrm{E}, 18-30 \mathrm{~km}$ and the respective latitude range as listed below) of the hotspots.

\begin{tabular}{|c|c|c|c|c|c|}
\hline Simulation & Abbreviation & Region & $\begin{array}{r}\operatorname{Min} / \max \mathrm{GWD}_{u} \\
\left(\mathrm{~m} \mathrm{~s}^{-1} \mathrm{~d}^{-1}\right)\end{array}$ & $\begin{array}{r}\operatorname{Min} / \max \mathrm{GWD}_{v} \\
\left(\mathrm{~m} \mathrm{~s}^{-1} \mathrm{~d}^{-1}\right)\end{array}$ & $\begin{array}{r}\operatorname{Min} / \max \mathrm{GWD}_{T} \\
\left(\mathrm{Kd}^{-1}\right)\end{array}$ \\
\hline Reference & Ref & & $-0.025 / 0.02$ & $-0.025 / 0.01$ & $-0.0006 / 0.01$ \\
\hline \multirow[t]{9}{*}{ Hotspots as 3-D box } & H1 & $27.5-52.5^{\circ} \mathrm{N}$ & -10 & -0.1 & 0.05 \\
\hline & $\mathrm{H} 2$ & $32.5-57.5^{\circ} \mathrm{N}$ & & & \\
\hline & $\mathrm{H} 3$ & $37.5-62.5^{\circ} \mathrm{N}$ & & & \\
\hline & $\mathrm{H} 4$ & $42.5-67.5^{\circ} \mathrm{N}$ & & & \\
\hline & H5 & $47.5-72.5^{\circ} \mathrm{N}$ & & & \\
\hline & H6 & $52.5-77.5^{\circ} \mathrm{N}$ & & & \\
\hline & $\mathrm{H} 7$ & $57.5-82.5^{\circ} \mathrm{N}$ & & & \\
\hline & $\mathrm{H} 8$ & $62.5-87.5^{\circ} \mathrm{N}$ & & & \\
\hline & & $\begin{array}{r}118.1-174.3^{\circ} \mathrm{E} \\
18-30 \mathrm{~km}\end{array}$ & & & \\
\hline Hotspots as 3-D Gaussian distribution & Gauss & & -13.1 & -0.13 & 0.065 \\
\hline
\end{tabular}

polar stratosphere. Above $35-40 \mathrm{~km}$, we observe a positive vertical wind anomaly for the $\mathrm{H} 1-\mathrm{H} 3$ simulations, i.e., the downward movement is reduced and leads to an adiabatic cooling anomaly. For most of the simulations, the negative anomaly in the lower part of the stratosphere is stronger than the positive anomaly above $40 \mathrm{~km}$, which fits with the distribution of the temperature anomalies. In case of the $\mathrm{H} 4$ and H5 simulations (Fig. 4d, e), the vertical wind anomalies do not fit with the temperature anomalies. We observe an increased downward movement in a region where the temperature weakly decreases.

\subsection{Influence on the polar vortex and anomalous SPWs}

From previous publications, it is already known that a warming (cooling) of the high-latitude stratosphere (mesosphere) and related changes in the dynamics are generally connected with PW activity. This leads us to the hypothesis that the main GWD enhancement effect is due to SPW modulation, and this will be investigated in this subsection. In Fig. 5, we show the geopotential height (as contour lines) and the zonal wind (using color coding) as a polar plot at $35 \mathrm{~km}$, i.e., $5 \mathrm{~km}$ above the region of GW forcing, for the H3 (Fig. 5a), H7 (Fig. 5b) and Ref simulations (Fig. 5c). The panels represent the last $30 \mathrm{~d}$ of analysis. The position of each $\mathrm{GW}$ hotspot is illustrated by the boxes, H1 (black) to H8 (violet), in Fig. 5c. The polar vortex of the Ref simulation is stable (not displaced or split) and located near the North Pole (Fig. 5c). Between 30 and $55^{\circ} \mathrm{N}$, the zonal wind of the Ref simulation is easterly in one part of the EA/NP region due to the Aleutian High (AH). This means that the GW forcing, which normally acts against the westerly zonal mean zonal wind, locally strengthens the zonal wind. Between 55 and $90^{\circ} \mathrm{N}$ there is a strong westerly wind between East Asia and Alaska; thus, the GW forcing there acts locally against the zonal wind. Most of the GW hotspots (H1-H5) are located in the transition zone between easterlies and westerlies; therefore, the southeastern (northwestern) part of each GW hotspot is located within the region of the easterlies (westerlies). The part of the GW forcing that is located in the easterlies (westerlies) decreases (increases) for the more northward-shifted GW hotspots (Fig. 5c). In the H3 simulation, half of the GW hotspot is located in the easterlies and the other half in the westerlies. The state of the polar vortex for the $\mathrm{H} 3$ simulation is presented in Fig. 5a. The AH completely disappears (the easterly wind ceases), and the center of the polar vortex is shifted towards Canada and Greenland. The polar vortex is comma-like in shape and slightly weaker and broader than the polar vortex of the Ref simulation. Thus, the H3 GW forcing has a destructive effect on the vortex circulation. This is in accordance with the results of the zonal wind differences (H3 - Ref) in Fig. 3c showing the displacement of the polar vortex edge to lower latitudes. With respect to the H7 GW hotspot (Fig. 5b), which is completely located in the westerlies, the polar vortex is less disturbed by the GW forcing and remains approximately in the same position as in the Ref simulation. The non-zonal part of the zonal wind field, which is mainly dominated by the SPW 1 , will interact with the local zonal wind anomaly induced by the localized GW forcing. As this zonal wind anomaly is localized in longitude, it may be decomposed into a spectrum of harmonics and can be assumed to be an additional wave interacting with the original zonal wind SPW 1. To examine this interaction between the original SPW 1 in the model and that induced by the local GW forcing, the difference in the SPW 1 zonal wind amplitude between each GW hotspot simulation $\mathrm{H} 1-\mathrm{H} 8$ and the Ref simulation is shown in Fig. 6. The position of each GW hotspot is illustrated by the red boxes. In the Ref simulation, the SPW 1 amplitude reaches a maximum at about $55 \mathrm{~km}$ between 30 and $40^{\circ} \mathrm{N}$ of more than $28 \mathrm{~m} \mathrm{~s}^{-1}$ and also 
(a)

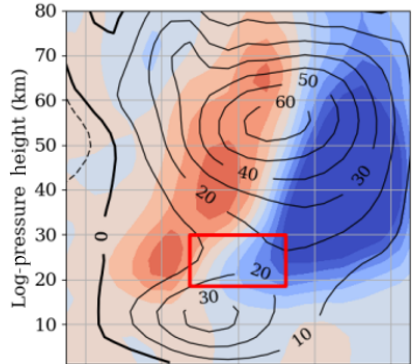

(e)

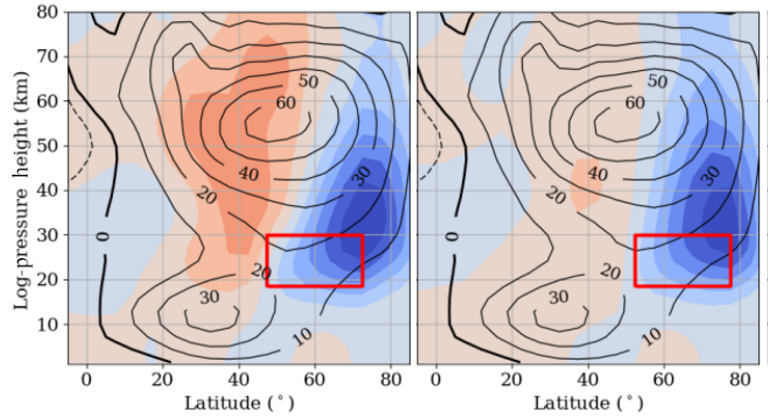

(c)

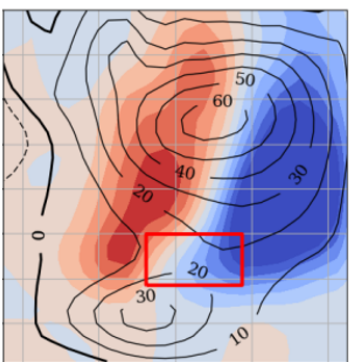

(f)
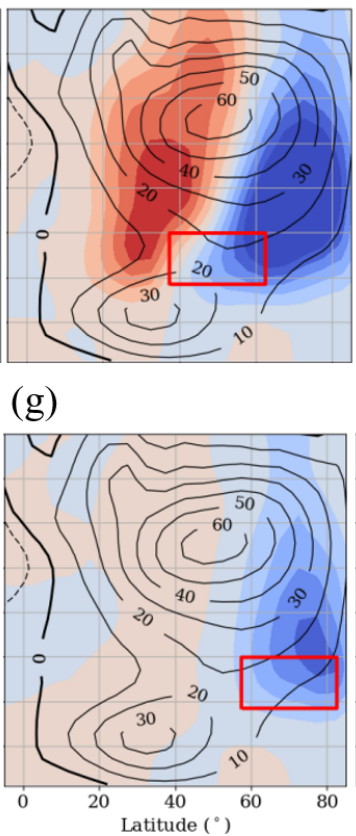

(g) (d)

(h)
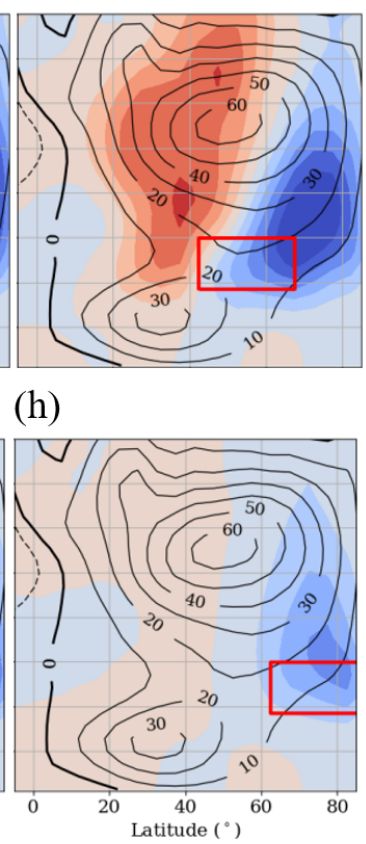

Figure 3. Zonal mean zonal wind difference between the H1-H8 simulations (a-h) and the reference simulation (H1-H8 - Ref). The colors indicate the difference between the simulations, and the contour lines show the zonal mean zonal wind of the reference simulation. Figures represent the last $30 \mathrm{~d}$ of the simulations. The position of each GW hotspot is represented by a red box.

(a)

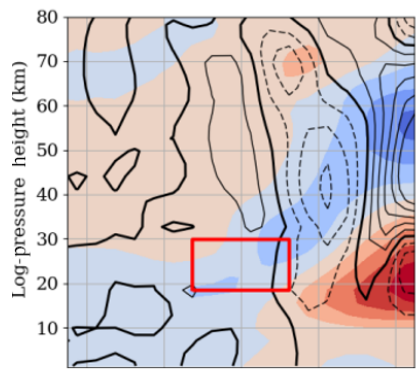

(e) (b)

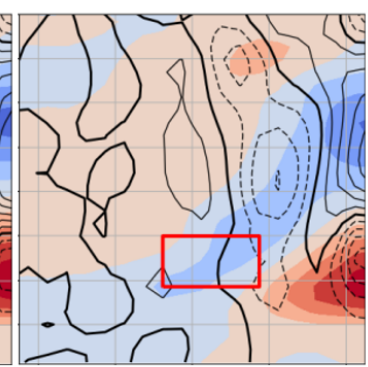

(f) (c)

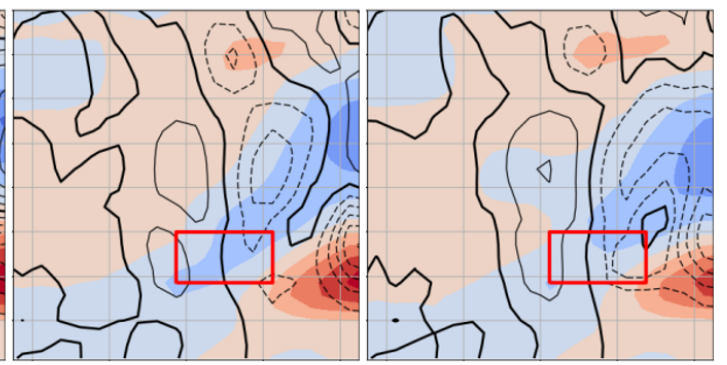

(g) (d)

(h)

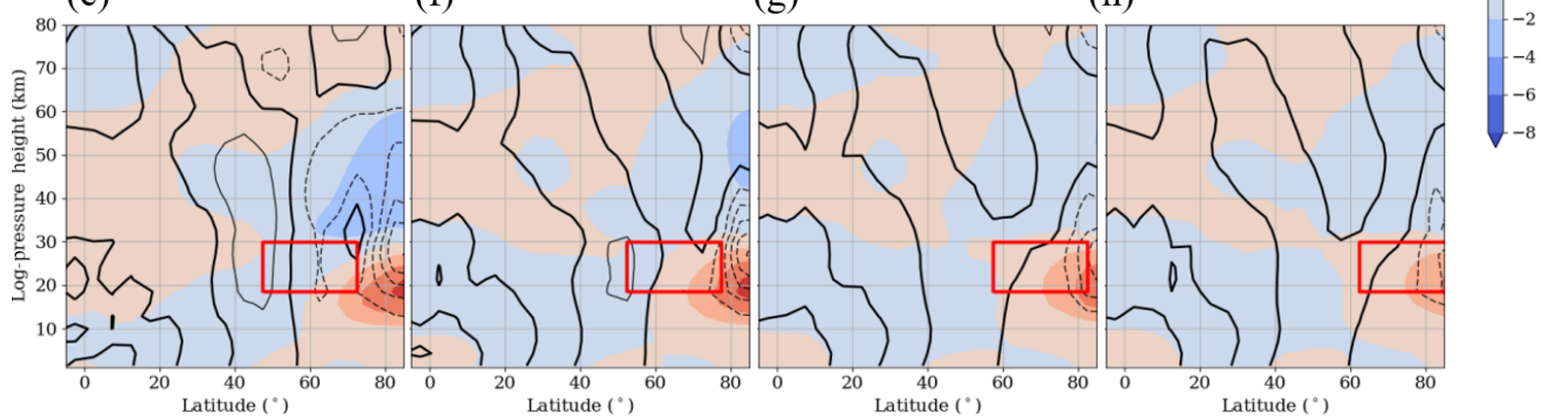

Figure 4. Zonal mean temperature and vertical wind difference between the H1-H8 simulations (a-h) and the reference simulation (H1$\mathrm{H} 8$ - Ref). The colors indicate the temperature difference between the simulations, and the contour lines show the vertical wind difference from \pm 0.0005 to $\pm 0.0025 \mathrm{~m} \mathrm{~s}^{-1}$ with increments of $0.0005 \mathrm{~m} \mathrm{~s}^{-1}$ and a thicker zero line. Negative (positive) values are shown using dashed (solid) lines. Figures represent the last $30 \mathrm{~d}$ of the simulations. The position of each $\mathrm{GW}$ hotspot is represented by a red box. 
(a) $37.5-62.5^{\circ} \mathrm{N}(\mathrm{H} 3)$

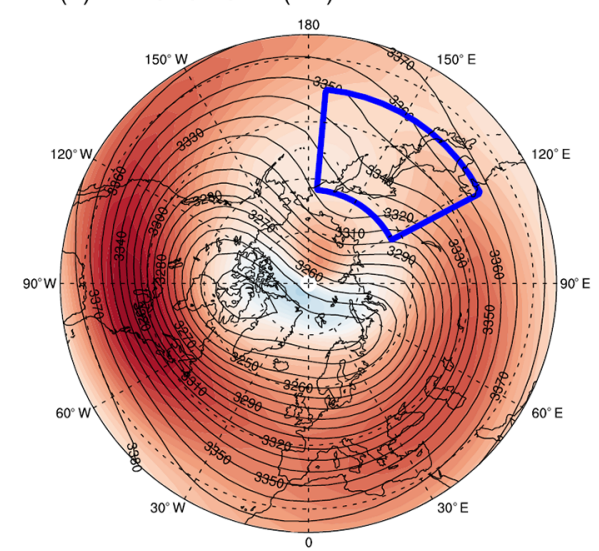

(b) $57.5-82.5^{\circ} \mathrm{N}(\mathrm{H} 7)$

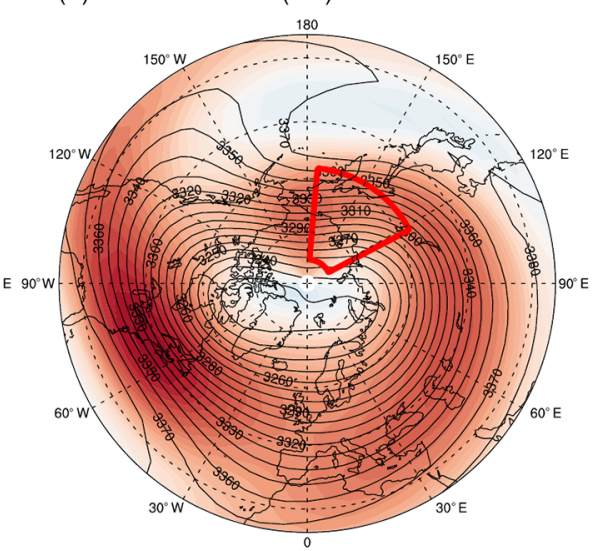

(c) Reference (Ref)

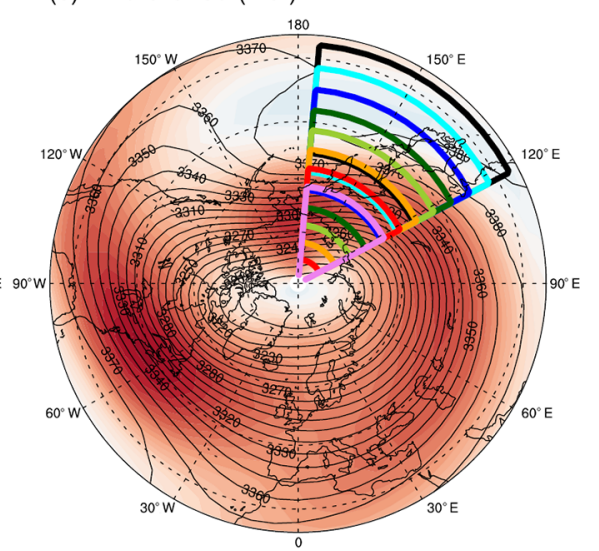

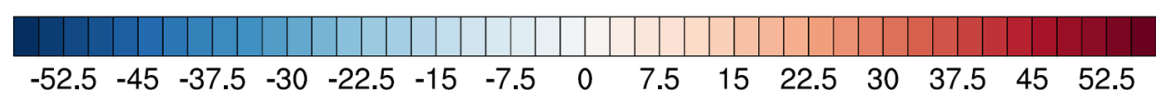

Figure 5. Zonal wind $\left(\mathrm{m} \mathrm{s}^{-1}\right)$ in color and geopotential height (gpdam) as contour lines north of $25^{\circ} \mathrm{N}$ at $35 \mathrm{~km} \mathrm{for} \mathrm{the} \mathrm{H} 3$ (a), $\mathrm{H} 7(\mathbf{b})$ and the Ref (c) simulations, representing the last $30 \mathrm{~d}$ of analysis. The boxes illustrate the position of each GW hotspot: H1 (in black) to H8 (in violet). The blue (a, c) (red, b, c) box refers to the H3 (H7) simulation.

in the polar stratosphere of about $20 \mathrm{~m} \mathrm{~s}^{-1}$. With respect to the $\mathrm{H} 1-\mathrm{H} 4$ simulations, in Fig. 6a-d the zonal wind SPW 1 amplitude differences are positive (negative) on the northern (southern) flank of the respective GW hotspot up to an altitude of about $60 \mathrm{~km}$. The negative (positive) SPW $1 \mathrm{am}-$ plitude anomaly increases (decreases) for more GW hotspots located further north. The strongest increase (decrease) in the SPW 1 amplitude can be observed in the H1 (H4) simulation, with more than $8 \mathrm{~m} \mathrm{~s}^{-1}\left(-8 \mathrm{~m} \mathrm{~s}^{-1}\right)$. By comparing the positive and negative SPW 1 amplitude anomalies of the $\mathrm{H} 1-\mathrm{H} 4$ simulations it can be seen that the positive anomaly is less pronounced, whereas the negative anomaly is more prevalent throughout the $\mathrm{NH}$, particularly around the stratopause. The decreasing SPW 1 amplitude indicates that fewer SPWs 1 are propagating into the middle atmosphere. Due to the decreasing SPW 1 activity at lower latitudes, fewer SPWs 1 are breaking in this region, i.e., the zonal mean zonal wind is less decelerated (as is shown in Fig. 3). Nonlocally, however, a localized destructive (constructive) superposition of the original SPWs 1 within the model and that of the GW forcing may decrease (increase) the SPW 1 amplitude at other heights/latitudes due to changes in PW propagation. This effect can be seen around $55^{\circ} \mathrm{N}$, where we observe an enhanced SPW 1 amplitude. It is strongest for the H1 GW hotspot and decreases for GW hotspots located further north. The suppressed upward propagation of SPWs 1 leads to an increase in the SPW 1 amplitude in this area. This positive SPW 1 amplitude anomaly corresponds to the decelerated zonal mean zonal wind in Fig. 3. This leads to the assumption that the GW forcing may locally increase or decrease the SPW 1 amplitude, but prevents the SPWs from propagating upwards into higher altitudes; hence, the SPW 1 amplitude mainly decreases in the stratosphere/mesosphere. Thus, the local GW forcing has a destructive effect on the circulation in the middle atmosphere.

We will verify this in Sect. 3.3 by analyzing the EliassenPalm flux. Owing to the suppression of SPW 1 propagation at midlatitudes, the SPWs may increasingly propagate via the polar region, which may explain the increased SPW 1 amplitude in the polar stratosphere north of $75^{\circ} \mathrm{N}$. Another positive SPW 1 amplitude anomaly can be observed in the midlatitudinal mesosphere above $60 \mathrm{~km}$, which may be induced by local instabilities generating new SPWs 1 . Both of these positive SPW 1 amplitude anomalies are strongest for the $\mathrm{H} 1$ simulation and once again decrease for northward-shifted GW hotspots. The SPW 1 amplitude anomalies for the four northernmost GW hotspots, H5-H8 in Fig. 6e-h, are small in comparison with the four southernmost GW hotspot simulations, which correspond to the observations in Sect. 3.1. Only for the H5 simulation (Fig. 6e) is the SPW 1 activity also strongly reduced at lower latitudes above $30 \mathrm{~km}$, as in the $\mathrm{H} 1-\mathrm{H} 4$ simulations.

To analyze the extent to which the GW forcing locally affects the SPWs of wave number 2 and 3, we compared the SPW 1 (Fig. 7a), 2 (Fig. 7b) and 3 (Fig. 7c) amplitude anomalies at $35 \mathrm{~km}$ northward of $0^{\circ} \mathrm{N}-\mathrm{S}$. The colors are the same as the colors used for the hotspots in Fig. 5. As previously discussed in Fig. 6, the SPW 1 amplitude locally increases at midlatitudes and in the polar region with a maximum of about $10 \mathrm{~m} \mathrm{~s}^{-1}$, with the maxima decreasing for northward-displaced GW hotspots. The negative anomaly, which is mainly dominant in the middle atmosphere, is located between 30 and $40^{\circ} \mathrm{N}$ as well as at $70^{\circ} \mathrm{N}$, with a minimum of more than $-10 \mathrm{~m} \mathrm{~s}^{-1}$. From the SPW 2 amplitude, it can be seen that the SPW 2 activity is weakened or reduced northward of $30^{\circ} \mathrm{N}$ by about $-6 \mathrm{~m} \mathrm{~s}^{-1}$ at the minimum. The 
(a)

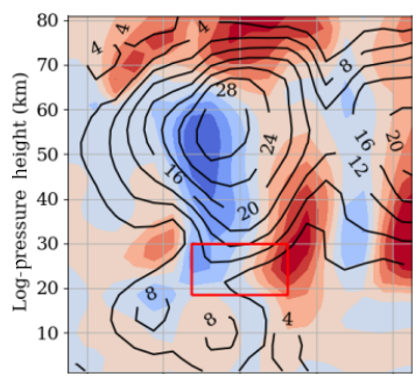

(e)

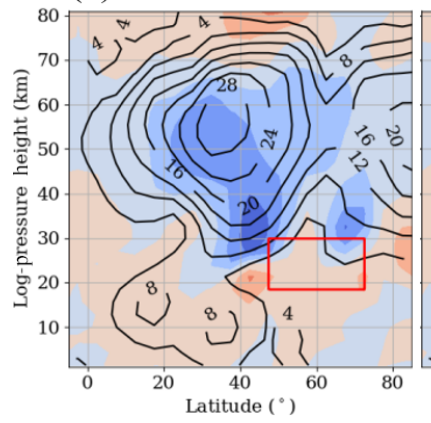

(b)

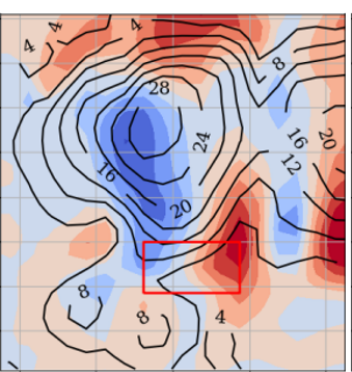

(f) (c)

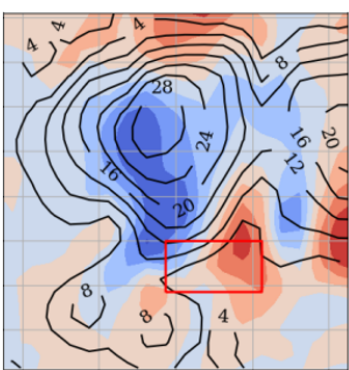

(g)

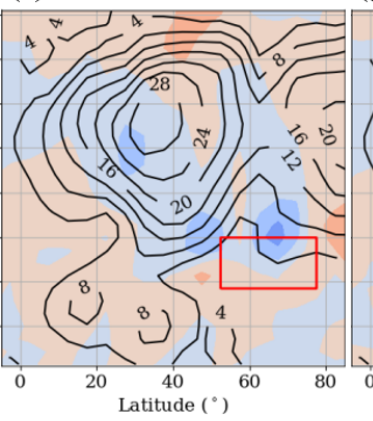

(g)

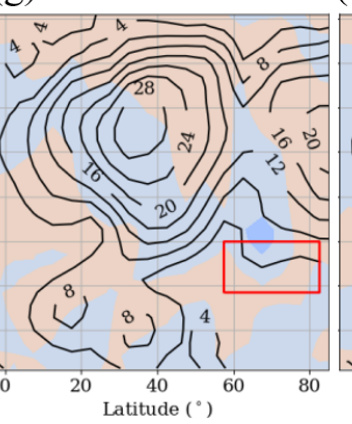

(d)

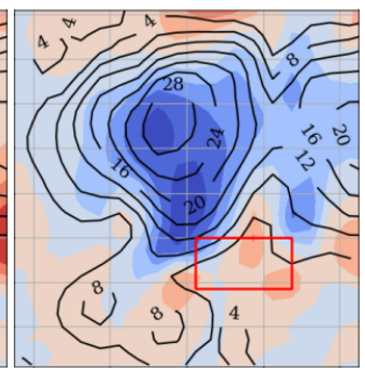

(h)

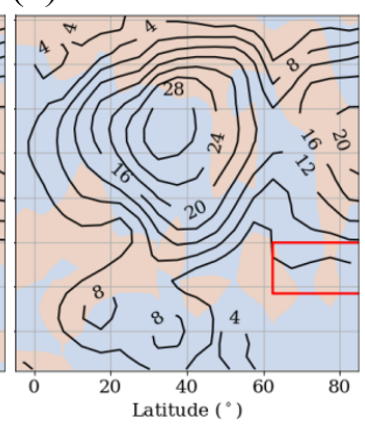

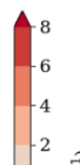

0 is 目

Figure 6. Zonal mean SPW 1 amplitude extracted from the zonal wind as the difference between the H1-H8 simulations (a-h) and the reference simulation. The colors indicate the difference between the simulations, and the contour lines show the zonal mean SPW 1 amplitude of the reference simulation. Figures represent the last $30 \mathrm{~d}$ of the simulations. The position of each $\mathrm{GW}$ hotspot is represented by a red box.

largest decrease can be observed for the southernmost GW hotspot, and the decrease becomes smaller for northwarddisplaced GW hotspots. Only at lower latitudes is the SPW 2 amplitude slightly increasing for those simulations. This is the case for the $\mathrm{H} 2, \mathrm{H} 3, \mathrm{H} 4$ and $\mathrm{H} 5$ simulation. The SPW 2 anomaly is negative (positive) in the regions where the SPW 1 anomaly is positive (negative). This leads to the assumption that just one of both SPWs (SPW 1 and SPW 2) can be dominant. By comparing the latitudinal distribution of the SPW 1 and 2 amplitude anomalies northward of $30^{\circ} \mathrm{N}$, it can be seen that they are similar when we neglect the scales. Both show a decrease in amplitude around 40 and $70^{\circ} \mathrm{N}$ and an increase in the midlatitudes and in the polar region. In comparison, the SPW 3 amplitude anomaly distribution (Fig. 7c) is slightly different, as the SPW 3 amplitude decreases at $20^{\circ} \mathrm{N}$ (not at $40^{\circ} \mathrm{N}$, as seen for the SPW 1 and 2 anomalies). However, as for the SPW 1 and 2 anomalies, an increase in the SPW 3 amplitude induced by the local GW hotspots can be observed in the midlatitudes with a maximum of about $2 \mathrm{~m} \mathrm{~s}^{-1}$. The largest increase in SPW 3 amplitude can be seen in the H1 simulation (southernmost GW hotspot), and the largest decrease is observed in the $\mathrm{H} 3$ simulation (observed Asian GW hotspot).

The suppression of SPWs, which is induced by the local GW forcing, might also be an effect partly induced by the shape of the GW hotspot, leading to a sharp transition zone between the unchanged and enhanced GW drag values. To prove that the shape of the GW hotspot partly leads to a sup- pression of SPWs, Fig. 8 shows the $\mathrm{H} 3$ amplitude anomaly from Fig. 7a and the corresponding Gauss simulation described in Sect. 2. The latitudinal distribution of the SPW 1 amplitude difference is still the same, showing the two local maxima at the midlatitudes and in the polar region and the two minima at 40 and $70^{\circ} \mathrm{N}$, although these two minima decreased from $-8 \mathrm{~m} \mathrm{~s}^{-1}$ for the 3-D box to $-4 \mathrm{~m} \mathrm{~s}^{-1}$ for the Gaussian distribution. Moreover, the maxima increased from about $4 \mathrm{~m} \mathrm{~s}^{-1}$ to more than $5 \mathrm{~m} \mathrm{~s}^{-1}$. Due to the stronger maximum GW drag in the Gaussian distribution the SPW 1 excitation is strengthened, which leads to the larger SPW 1 amplitudes at midlatitudes. The smoothly decreasing GW drag forcing towards lower and higher latitudes only slightly reduces the suppression of SPW 1 around 40 and $70^{\circ} \mathrm{N}$. The mean wind and temperatures are also only weakly affected if we replace the box-like forcing with one with a Gaussian shape (not shown here). Thus, the GW hotspot itself leads to essential changes in the dynamics, suppressing the SPW propagation and decreasing the SPW 1 activity in the middle atmosphere.

To sum up the influence on the polar vortex, in Sect. 3.1 we observed a slight warming of the lower stratosphere and a decreasing west wind at middle to high latitudes, which indicates a weakening of the polar vortex as a consequence of the GW drag enhancement. Thus, the stability of the polar vortex not only depends on the PW activity but also on the interplay or nonlinear interaction of GW and PW forcings. The anomalous SPW forcing and the suppression of SPW 

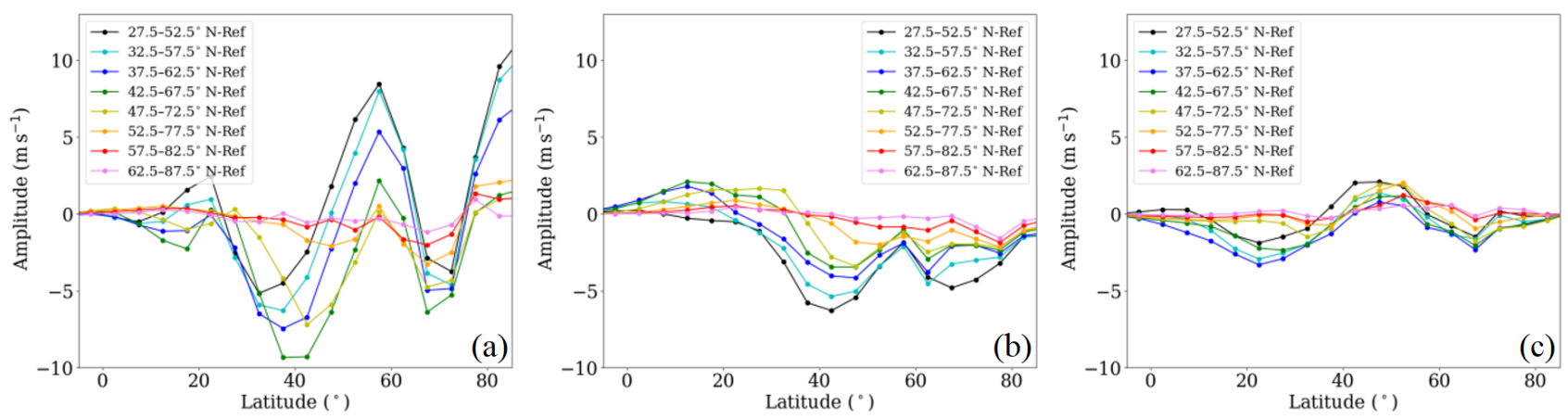

Figure 7. Zonal mean SPW 1 (a), SPW 2 (b) and SPW 3 (c) amplitudes as the difference between the H1-H8 simulations and the Ref simulation at $35 \mathrm{~km}$ for the last $30 \mathrm{~d}$ of the simulations extracted from the zonal wind.

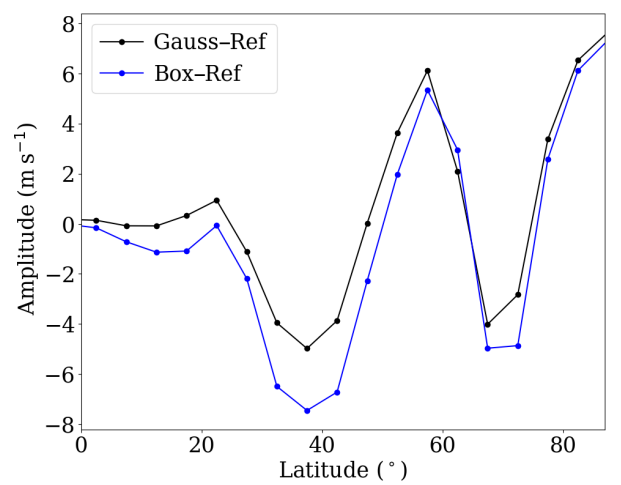

Figure 8. SPW 1 amplitude as the difference between the $\mathrm{H} 3$ simulation and the reference simulation (blue line) and the Gauss distribution and the reference simulation (black line) at $35 \mathrm{~km}$ for the last $30 \mathrm{~d}$ extracted from the zonal wind.

propagation show that the GW drag can play an important role in preconditioning the polar vortex (see next section).

\subsection{Propagation conditions for SPWs}

To establish the extent to which the SPW propagation is affected by the local GW forcing, the EP fluxes and their divergence for SPW 1 were calculated. The results of the Ref simulation are presented in Fig. 9a. The arrows show the direction of propagation, the color of the arrows represents the strength of the EP flux not normalized by the density, and the grey areas and the grey contour lines represent the EP divergence, showing the direction in which the zonal mean flow is accelerated. A negative (positive) EP divergence is illustrated by the dashed (solid) lines. The arrows were replaced by dots when the amplitude difference was smaller than $1 \%$ of the maximum EP flux amplitude difference. The waves mainly develop in the middle and higher latitudes, and from there they mainly propagate towards the equatorial stratosphere/stratopause and, to a much lesser degree, to the polar stratosphere. That the waves are really propagating upwards can be seen by means of the increasing amplitudes of the
EP fluxes. The maximum EP flux amplitudes of more than $1.4 \mathrm{~m}^{2} \mathrm{~s}^{-2}$ are reached between 50 and $60^{\circ} \mathrm{N}$ at an altitude of about $60 \mathrm{~km}$, which corresponds to the height of the SPW 1 amplitude maximum in Fig. If of the Ref simulation.

In Fig. 10 the difference in the EP flux and its divergence between the H1-H8 simulations and the Ref simulation is shown. The position of each GW hotspot is again illustrated by the red boxes. In the $\mathrm{H} 1$ and $\mathrm{H} 2$ simulations (Fig. 10a-b) more SPWs 1 propagate into the polar stratosphere. These SPWs 1 are partly coming from the midlatitudes but most of them are directly generated in the polar region, where we observe a source of SPWs 1 (enhanced positive EP divergence at $70^{\circ} \mathrm{N}$ between 20 and $30 \mathrm{~km}$ ). This positive EP divergence anomaly corresponds to the increased SPW 1 amplitude in the polar region in Fig. 7. Above this positive EP divergence anomaly an enhanced negative EP divergence is seen (from the northern flank of the GW hotspot up to $60 \mathrm{~km}$ tilted towards the north with increasing height), which means that the SPWs 1, which propagate via the Arctic stratosphere, break in this region. This leads to the deceleration of the zonal mean zonal wind in the middle and higher latitudes, as previously discussed in Fig. 3. The negative EP divergence anomaly can also be seen in the H3-H5 simulations (Fig. 10c-e). This is the reason why the polar vortex is mainly disturbed by these GW hotspots (H1-H5). The negative EP divergence is strongest for the $\mathrm{H} 1$ simulation, which also exhibits the strongest increase in SPW 1 amplitude in the polar region. Furthermore, the H1-H5 simulations show a strong decrease in the EP flux amplitude (blue arrows) between 40 and $70 \mathrm{~km}$ and between 20 and $80^{\circ} \mathrm{N}$, which means that fewer SPWs 1 are propagating into the middle atmosphere. As a consequence, fewer SPWs 1 break in this region, leading to a positive EP divergence anomaly. This result corresponds to the decreasing SPW 1 amplitude (Fig. 7) and the increasing zonal mean zonal wind (Fig. 3) at lower latitudes. The effect is strongest for the $\mathrm{H} 4$ simulation, which also shows the strongest decrease in SPW 1 amplitudes. Between 40 and $70^{\circ} \mathrm{N}$ around $70 \mathrm{~km}$ we observe another source of SPWs 1, which propagate into the mesosphere, where these waves break (strongly negative EP divergence above 

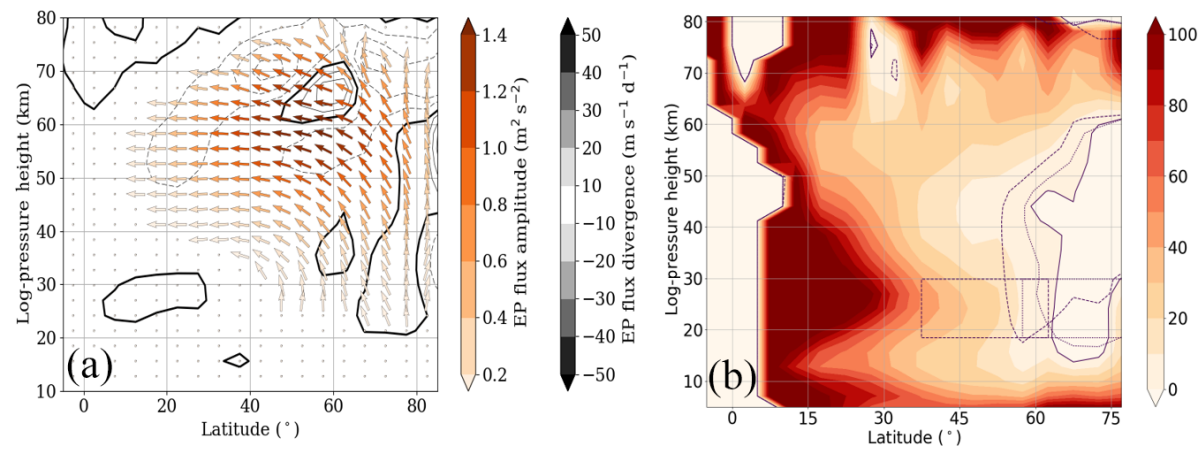

Figure 9. Zonal mean EP flux of SPW 1 of the Ref simulation (a). Contour lines show the EP flux divergence, and dashed lines denote negative EP flux divergence. The refractive index for SPW 1 for the Ref simulation (b) with a thicker zero line. The position of the H3 (H7) GW hotspot and the respective zero line is represented by the dashed (dotted) violet line. Both panels represent the last $30 \mathrm{~d}$ of the simulation.

(a)

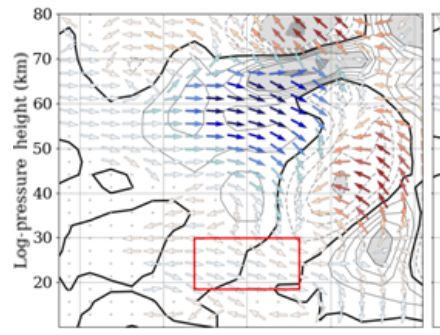

(e) (b)

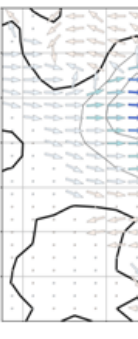

(f) (c) (d)

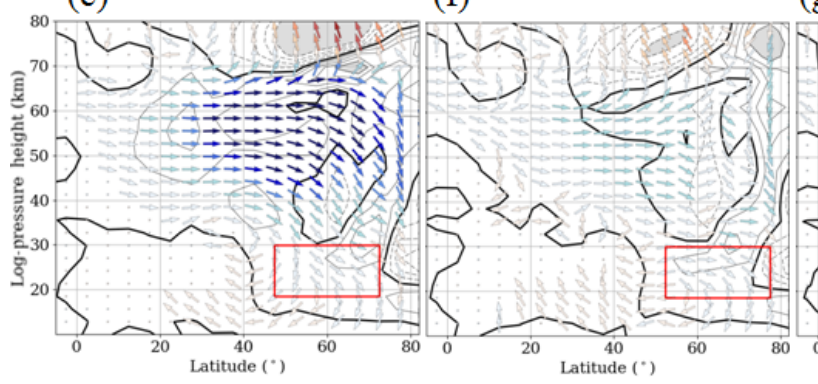

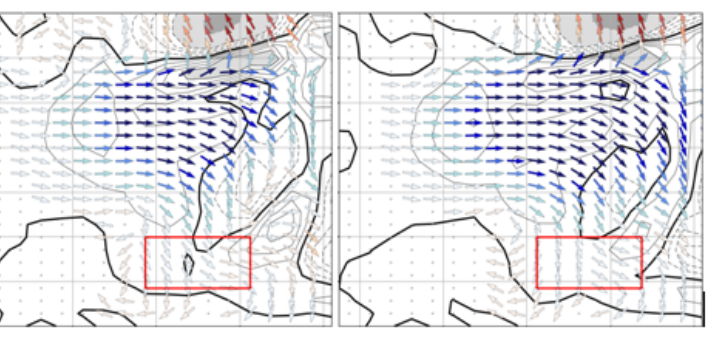

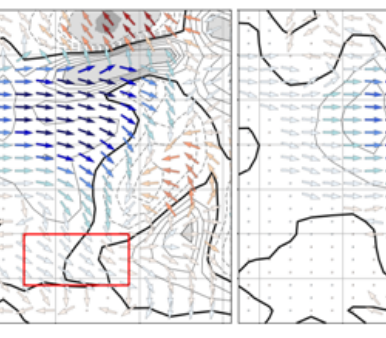

(g)

(h)

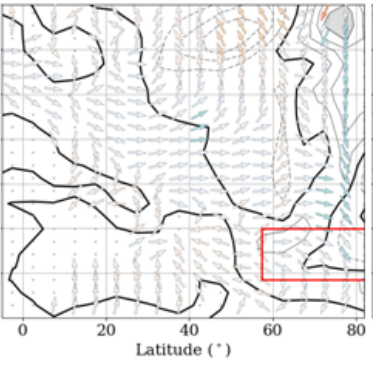

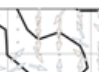

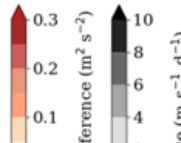

0.0

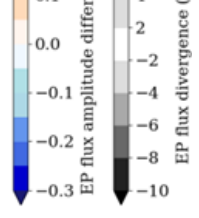

Figure 10. Zonal mean EP flux (arrows) and divergence (isolines and shaded areas; dashed lines show negative values) of SPW 1. Shown is the difference between all $\mathrm{H} 1-\mathrm{H} 8$ simulations (a-h) and the reference simulation (H1-H8 - Ref) representing the last $30 \mathrm{~d}$ of the simulations.

the positive EP divergence) due to the reversed wind conditions. The mesospheric EP flux in the H1-H5 simulations corresponds to the observed enhanced SPW 1 amplitude in the mesosphere in Fig. 7. Referring to the enhanced SPW 1 around $55^{\circ} \mathrm{N}$ of the GW hotspots, no enhanced EP flux can be observed in the respective region. However, the arrows of the EP flux anomalies are pointing towards this area of enhanced SPW 1 amplitude. In the H6-H8 GW hotspot simulations (Fig. 10f-h) no large differences in EP flux and divergence occur, which correspond to the small SPW 1 amplitude and the zonal mean zonal wind differences in Figs. 7 and 3 .

To explain why SPWs 1 do not propagate at higher latitudes, the refractive index (Matsuno, 1971; Andrews et al., 1987), multiplied by the square of the Earth's radius $a^{2}$, is also shown in Fig. 9b. The refractive index is highly dependent on the meridional potential vorticity gradient $\left(q_{y}\right)$ and on the zonal mean zonal wind conditions ( $\mathrm{Li}$ et al., 2007). White regions in Fig. 9b indicate a negative refractive index, which means that the waves cannot propagate in these regions. In the reddish regions wave propagation is possible. Due to the predominating westerly wind in the $\mathrm{NH}$, the refractive index is mostly positive; therefore, SPWs 1 are able to propagate predominantly upward and towards the Equator. Towards the midlatitudes and the polar region the refractive index decreases due to the increasing zonal mean zonal wind. The polar region (north of $60^{\circ} \mathrm{N}$ ) is the only region in the NH with a negative refractive index. This is because the polar vortex is a strong closed system, which repels most of the waves. To establish the extent to which the refractive 

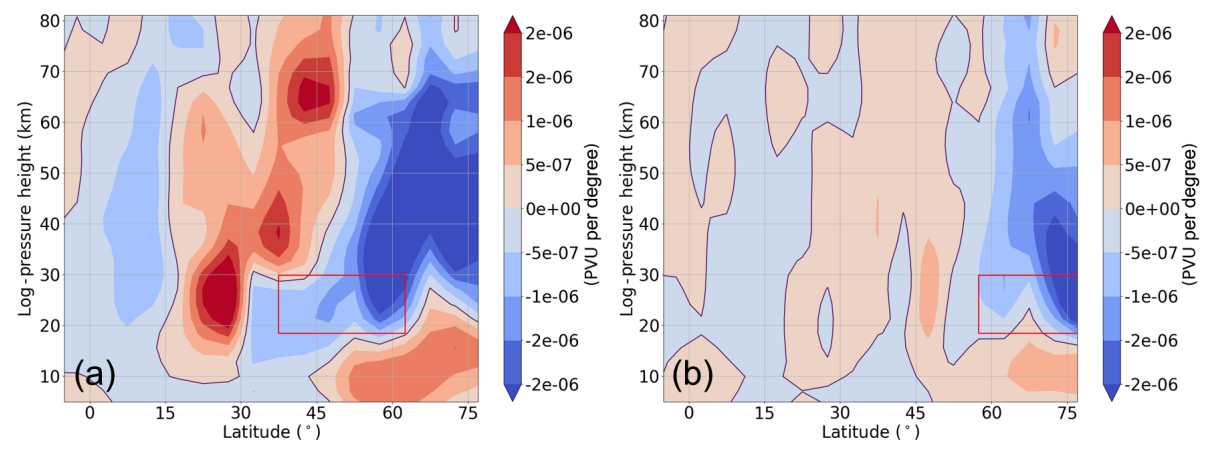

Figure 11. Meridional potential vorticity gradient difference between the respective H3 (a)and H7 (b) simulations and the reference simulation, representing the last $30 \mathrm{~d}$ of the simulations. The positions of the $\mathrm{H} 3$ and $\mathrm{H} 7 \mathrm{GW}$ hotspots are represented by red boxes.

index changes after the implementation of the GW forcing, the position of the $\mathrm{H} 3$ (dashed violet line) and the $\mathrm{H} 7$ (dotted violet line) GW hotspot and the respective zero line of the refractive index were added to Fig. 9b. In the $\mathrm{H} 3$ simulation, the zero line is higher in the polar region than in the Ref simulation. Thus, the refractive index increases (becomes more positive) in the polar region below $30 \mathrm{~km}$, which corresponds to the enhanced SPW 1 propagation and SPW 1 amplitude in the same region. The zero line of the $\mathrm{H} 7$ simulation is almost at the same height as that of the Ref simulation; thus, we do not observe huge changes in the Arctic. While the zero line of the Ref simulation is limited to the regions north of $60^{\circ} \mathrm{N}$, the zero line of the $\mathrm{H} 3(\mathrm{H} 7)$ simulation is located around $50^{\circ} \mathrm{N}$ $\left(57^{\circ} \mathrm{N}\right)$. Based on the EP flux distribution of the Ref simulation in Fig. 9a, we already know that the SPWs 1 mainly propagate from the midlatitudes (between 50 and $60^{\circ} \mathrm{N}$ ) into the middle atmosphere. Due to the negative refractive index in this region, the SPWs 1 in the $\mathrm{H} 3$ and $\mathrm{H} 7$ simulations are no longer able to propagate upwards, meaning that the SPW 1 EP flux and amplitude decrease. Thus, the major branch of SPW 1 propagation is interrupted by the local GW forcing. To check if there are local instabilities leading to the SPW 1 sources in the polar region and in the lower mesosphere, the $q_{y}$ differences between the $\mathrm{H} 3(\mathrm{H} 7)$ and the Ref simulation are shown in Fig. 11. The $q_{y}$ is given in potential vorticity units (PVU) per degree. The positions of the $\mathrm{H} 3$ and H7 GW hotspot are illustrated using red boxes. Due to the increasing (decreasing) zonal mean zonal wind at lower (higher) latitudes, the $q_{y}$, which normally increases towards higher latitudes, is reversed northward of $30^{\circ} \mathrm{N}$. We observe a negative $q_{y}$ anomaly, which is tilted towards the north with increasing height. Northward of $45^{\circ} \mathrm{N}$ up to $20 \mathrm{~km}$ the $q_{y}$ anomaly reverses again and becomes positive. These local reversals of the $q_{y}$, which are a necessary condition for baroclinic instability (Charney and Stern, 1962), can lead to the SPW 1 sources and positive EP divergences in the respective regions.

\section{Conclusions}

The sensitivity study regarding the effect of local GW hotspots in the stratosphere from lower to higher latitudes in a specific longitude range (between 120 and $170^{\circ} \mathrm{E}$ ) shows that GW hotspots south of $50^{\circ} \mathrm{N}$ lead to a negative refractive index at midlatitudes, which prevents the SPWs from propagating upwards. Thus, fewer SPWs 1 are breaking in the middle atmosphere corresponding to the decreasing SPW 1 amplitude at lower latitudes connected with an increasing zonal mean zonal wind. Thus, the polar vortex is shifted towards lower latitudes but remains very strong (Baldwin and Holton, 1988), which additionally leads to a suppression of SPWs according to the Charney-Drazin criterion (Charney and Drazin, 1961). The displacement of the polar vortex induced by breaking SPWs 1 causes an increase in the refractive index in the polar stratosphere (Karami et al., 2016); thus, the SPWs 1 originating at midlatitudes partly propagate via the polar region into the middle atmosphere. Apart from these SPWs 1, additional SPWs 1 propagating upwards are directly generated in the Arctic owing to local baroclinic instability; one indication of this is the reversal of the $q_{y}$ (Charney and Stern, 1962; Garcia, 1991). For this reason we observe an enhanced EP flux and, thus, an enhanced SPW 1 amplitude in the polar region. These SPWs 1 break around $50 \mathrm{~km}$ between 50 and $80^{\circ} \mathrm{N}$ and lead to an enhanced negative EP divergence connected with a decrease in zonal mean zonal wind at higher latitudes. In the lower mesosphere between 40 and $70^{\circ} \mathrm{N}$ there is a second source of SPWs 1 (positive EP divergence) in addition to local baroclinic instabilities (reversal of the $q_{y}$ ) (Smith, 2003; Lieberman et al., 2013; Matthias and Ern, 2018). As a consequence, the EP flux and the SPW 1 amplitude are enhanced between 70 and $80 \mathrm{~km}$, right above the positive EP divergence anomaly. Based on the SPW 1 amplitude extracted from the zonal GW drag (not shown here) it was clear that each of the GW hotspots leads to a forcing of SPWs 1, but in some regions northward of $50^{\circ} \mathrm{N}$ this forcing is ineffective because the waves cannot propagate or are eliminated by destructive interference. The refractive index, which is highly dependent on the zonal mean zonal 
wind conditions, shows negative values in the polar region for the Ref simulation. Thus, if we implement a GW forcing directly in this region it has no impact on the middle atmosphere as SPWs cannot propagate. If we provoke preconditioning of the polar vortex by first implementing, for example, the H1 GW hotspot and then adding one of the H6-H8 GW hotspots, the GW hotspots near the polar region would have a larger impact on the dynamics of the middle atmosphere.

Based on the results of the sensitivity study, we see that a local GW forcing can lead to a weakening (warming of the lower stratosphere) and slight displacement of the polar vortex at high latitudes, which is highly dependent on the strength (Š́cha et al., 2016) and the zonal distribution of the forcing (this study). Usually, it is assumed that preconditioning of the polar vortex is mainly driven by enhanced PW activity (Labitzke, 1981). However, there are also several indications based on satellite observations (Ern et al., 2016) and reanalysis data (Albers and Birner, 2014) which show that the GW drag and the absolute GW momentum flux is enhanced (reduced) in the stratosphere right before (after) SSWs. Albers and Birner (2014) analyzed the total wave forcing from the Japanese Meteorological Agency and Central Research Institute of Electrical Power Industry 25year Reanalysis (JRA-25) data before SSW events and found that up to $70 \%$ of the total drag is induced by orographic GWs. Ern et al. (2016) directly derived the GW drag and absolute momentum fluxes from HIRDLS and SABER temperatures and found that both parameters are enhanced before and around the central day of a SSW (strong polar jet), and they are reduced when the zonal wind is weak (after SSW). Because we kept the GW drag forcing constant throughout the experiment we cannot evaluate nonlinear effects, which would possibly reduce the GW drag connected with the displacement of the polar vortex. Furthermore, we have a fixed GW source distribution, meaning that no additional GWs are generated owing to changes in the tropospheric circulation. Nevertheless, on the basis of the zonal and meridional GW flux, which changes according to the propagation conditions, we analyzed the absolute horizontal GW momentum flux (not shown here). In this case, we also observe a reduction in the GW flux when the zonal mean zonal wind decreases at high latitudes. However, this effect is not very pronounced in our experiments, as the zonal mean zonal wind differences are much smaller than during a real SSW event. We only observe zonal mean zonal wind differences of about $-10 \mathrm{~m} \mathrm{~s}^{-1}$, which do not lead to a reversal of the zonal mean zonal wind or to subsequent significant background changes that may strongly influence the GW propagation.
Another interesting aspect is that different local GW forcing shapes do not have strong effects on the circulation. In spite of the Gaussian-smoothed boundaries, only negligible changes can be observed in the dynamics and SPW development, which are mainly due to the varying GW drag in the 3-D Gaussian distribution, leading to larger (smaller) effects when the Gaussian distribution reaches a maximum (minimum).

Comparing the positions of these simulated GW hotspots with measurements (e.g., Hoffmann et al., 2013), it is clear that at least some of the latitudinally shifted GW hotspots are not very realistic; therefore, our experiments should only be considered as a qualitative sensitivity study. Regarding orography, there are no obvious sources of orography when we displace the GW hotspot latitudinally. However, some of the GW hotspots can connect to jet exit regions on a purely hypothetical basis. To make the study more realistic, the next step would be to analyze the effect of a longitudinally shifted hotspot (fixed latitude range between 30 and $60^{\circ} \mathrm{N}$ ), as observations and GCM experiments have shown their existence (listed in the introduction). In this latitude range GW hotspots like the Himalayan region, the Alps or the Rocky mountains are included in the experiments. Also, the interaction of two or more GW hotspots is one of our focuses and will provide more insight into the effect of a localized GW forcing, which may be also important for the development of new GW parameterizations.

Code availability. MUAM model code is available from the corresponding author upon request. 


\section{Appendix A}

(a)

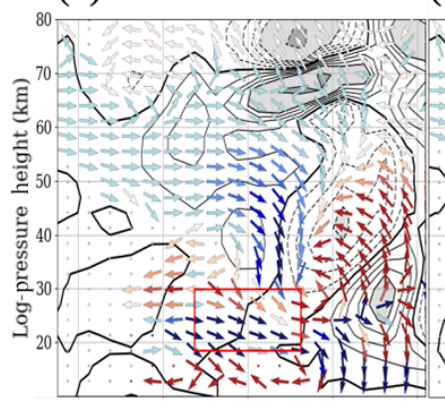

(e)

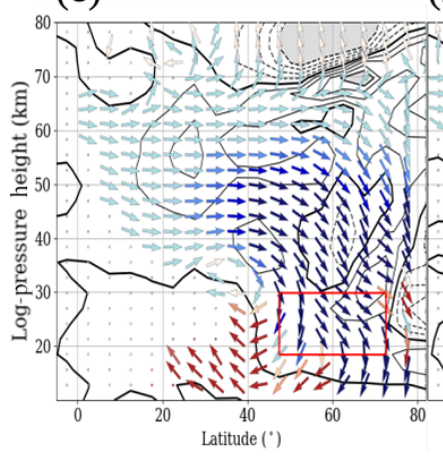

(b)

(c)

(d)

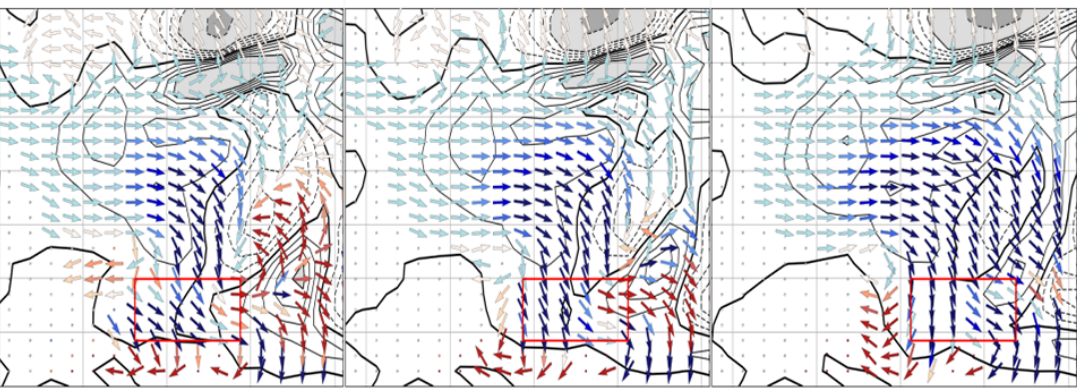

(f)

(g)

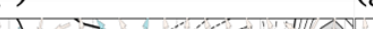

(h)

(g)
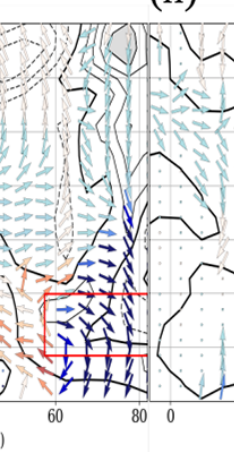

Figure A1. Zonal mean EP flux (arrows) and divergence (isolines and shaded areas; dashed lines show negative values) of SPW 1. Shown is the difference between all $\mathrm{H} 1-\mathrm{H} 8$ (a-h) simulations and the reference simulation (H1-H8 - Ref) representing the last $30 \mathrm{~d}$ of the simulations. The EP flux is weighted by the density. 
Author contributions. NS performed the MUAM model simulations and drafted the first version of the paper. PŠ provided the GW potential energy distributions. PŠ, PP, AK and CJ actively contributed to the discussions and to writing the paper.

Competing interests. Christoph Jacobi is one of the editors in chief of Annales Geophysicae. The authors declare that there are no conflicts of interest.

Special issue statement. This article is part of the special issue "Vertical coupling in the atmosphere-ionosphere system". It is a result of the 7th Vertical coupling workshop, Potsdam, Germany, 2-6 July 2018.

Acknowledgements. ERA-Interim reanalysis data were provided by ECMWF via the following website: https://www.ecmwf. int/en/forecasts/datasets/browse-reanalysis-datasets (last access: 27 June 2019)

Financial support. This research has been supported by the Deutsche Forschungsgemeinschaft (DFG; grant no. JA836/32-1) and by GA CR (grant no. 16-01562J). Petr Pišoft and Aleš Kuchař were supported by GA CR (grant nos. 16-01562J and 18-01625S). Petr Šácha was supported by GA CR (grant nos. 16-01562J and 18-01625S) and by the Government of Spain (grant no. CGL201571575-P) as well as via a postdoctoral grant from the Xunta de Galicia (grant no. ED481B 2018/103) in the later stages of the paper preparation. The EPhysLab is supported through the European Regional Development Fund (ERDF).

Review statement. This paper was edited by Kathrin Baumgarten and reviewed by two anonymous referees.

\section{References}

Albers, J. R. and Birner, T.: Vortex Preconditioning due to Planetary and Gravity Waves prior to Sudden Stratospheric Warmings, J. Atmos. Sci., 71, 4028-4054, https://doi.org/10.1175/JAS-D-140026.1, 2014

Alexander, P., Luna, D., Llamedo, P., and de la Torre, A.: A gravity waves study close to the Andes mountains in Patagonia and Antarctica with GPS radio occultation observations, Ann. Geophys., 28, 587-595, https://doi.org/10.5194/angeo-28-587-2010, 2010.

Andrews, D. G., Holton, J. R., and Leovy, C. B.: Middle Atmosphere Dynamics, ISBN 0-12-058576-6, Academic Press, San Diego, 1987.

Baldwin, M. P. and Holton, J. R.: Climatology of the stratospheric polar vortex and planetary wave breaking, J. Atmos. Sci., 45, 1123-1142, https://doi.org/10.1175/15200469(1988)045<1123:COTSPV>2.0.CO;2, 1988.
Charney, J. G. and Drazin, P. G.: Propagation of planetary-scale disturbances from the lower into the upper atmosphere, J. Geophys. Res., 66, 83-109, https://doi.org/10.1029/JZ066i001p00083, 1961.

Charney, J. G. and Stern, M. E.: On the Stability of Internal Baroclinic Jets in a Rotating Atmosphere, J. Atmos. Sci., 19, 159-172, https://doi.org/10.1175/15200469(1962)019<0159:OTSOIB>2.0.CO;2, 1962.

Costantino, L., Heinrich, P., Mzé, N., and Hauchecorne, A.: Convective gravity wave propagation and breaking in the stratosphere: comparison between WRF model simulations and lidar data, Ann. Geophys., 33, 1155-1171, https://doi.org/10.5194/angeo-33-1155-2015, 2015.

Dee, D. P., Uppala, S. M., Simmons, A. J., Berrisford, P., Poli, P., Kobayashi, S., Andrae, U., Balmaseda, M. A., Balsamo, G., Bauer, P., Bechtold, P., Beljaars, A. C. M., van de Berg, L., Bidlot, J., Bormann, N., Delsol, C., Dragani, R., Fuentes, M., Geer, A. J., Haimberger, L., Healy, S. B., Hersbach, H., Hólm, E. V., Isaksen, L., Kallberg, P., Köhler, M., Matricardi, M., McNally, A. P., Monge-Sanz, B. M., Morcrette, J.-J., Park, B.-K., Peubey, C., de Rosnay, P., Tavolato, C., Thépaut, J.-N., and Vitart, F.: The ERA-Interim reanalysis: configuration and performance of the data assimilation system, Q. J. Roy. Meteor. Soc., 137, 553-597, https://doi.org/10.1002/qj.828, 2011.

Douville, H.: Stratospheric polar vortex influence on Northern Hemisphere winter climate variability, Geophys. Res. Lett., 36, L18703, https://doi.org/10.1029/2009GL039334, 2009.

Ern, M. and Preusse, P.: Gravity wave momentum flux spectra observed from satellite in the summertime subtropics: Implications for global modeling, Geophys. Res. Lett., 39, L15810, https://doi.org/10.1029/2012GL052659, 2012.

Ern, M., Preusse, P., Alexander, M. J., and Warner, C. D.: Absolute values of gravity wave momentum flux derived from satellite data, J. Geophys. Res., 109, D20103, https://doi.org/10.1029/2004JD004752, 2004.

Ern, M., Ploeger, F., Preusse, P., Gille, J. C., Gray, L. J., Kalisch, S., Mlynczak, M. G., Russell III, J. M. R., and Riese, M.: Interaction of gravity waves with the QBO: A satellite perspective, J. Geophys. Res.-Atmos., 119, 2329-2355, https://doi.org/10.1002/2013JD020731, 2014.

Ern, M., Trinh, Q. T., Kaufmann, M., Krisch, I., Preusse, P., Ungermann, J., Zhu, Y., Gille, J. C., Mlynczak, M. G., Russell III, J. M., Schwartz, M. J., and Riese, M.: Satellite observations of middle atmosphere gravity wave absolute momentum flux and of its vertical gradient during recent stratospheric warmings, Atmos. Chem. Phys., 16, 9983-10019, https://doi.org/10.5194/acp16-9983-2016, 2016

Fleming, E. L., Chandra, S., Barnett, J. J., and Corney, M.: Zonal mean temperature, pressure, zonal wind and geopotential height as functions of latitude, Adv. Space Res., 10, 11-59, https://doi.org/10.1016/0273-1177(90)90386-E, 1988.

Fomichev, V. I. and Shved, G. M.: Parameterization of the radiative flux divergence in the $9.6 \mu \mathrm{m} \mathrm{O} 3$ band, J. Atmos. Terr. Phys., 47, 1037-1049, https://doi.org/10.1016/0021-9169(85)90021-2, 1985.

Fomichev, V. I., Blanchet, J.-P., and Turner, D. S.: Matrix parameterization of the $15 \mathrm{~mm} \mathrm{CO}_{2}$ band cooling in the middle and upper atmosphere for variable $\mathrm{CO}_{2}$ concentration, J. Geophys. Res. 103, 11505-11528, https://doi.org/10.1029/98JD00799, 1998. 
Fritts, D. C. and Alexander, M. J.: Gravity wave dynamics and effects in the middle atmosphere, Rev. Geophys., 41, 1003, https://doi.org/10.1029/2001RG000106, 2003.

Fröhlich, K., Pogoreltsev, A., and Jacobi, C.: Numerical simulation of tides, Rossby and Kelvin waves with the COMMA-LIM model, Adv. Space Res., 32, 863-868, https://doi.org/10.1016/S0273-1177(03)00416-2, 2003a.

Fröhlich, K., Pogoreltsev, A., and Jacobi, C.: The 48-layer COMMA-LIM model, Rep. Inst. Meteorol. Univ. Leipzig, 30, 157-185, available at: http://nbn-resolving.de/urn:nbn:de:bsz: 15-qucosa-217766 (last access: 27 June 2019), 2003 b.

Fröhlich, K., Schmidt, T., Ern, M., Preusse, P., de la Torre, A., Wickert, J., and Jacobi, C.: The global distribution of gravity wave energy in the lower stratosphere derived from GPS data and gravity wave modelling: attempt and challenges, J. Atmos. Sol.-Terr. Phy., 69, 2238-2248, https://doi.org/10.1016/j.jastp.2007.07.005, 2007.

Garcia, R. R.: Parameterization of planetary wave breaking in the middle atmosphere, J. Atmos. Sci., 48, 1405-1419, https://doi.org/10.1175/15200469(1991)048<1405:POPWBI>2.0.CO;2, 1991.

Hierro, R., Steiner, A. K., de la Torre, A., Alexander, P., Llamedo, P., and Cremades, P.: Orographic and convective gravity waves above the Alps and Andes Mountains during GPS radio occultation events - a case study, Atmos. Meas. Tech., 11, 3523-3539, https://doi.org/10.5194/amt-11-3523-2018, 2018.

Hoffmann, L., Xue, X., and Alexander, M. J.: A global view of stratospheric gravity wave hotspots located with Atmospheric Infrared Sounder observations, J. Geophys. Res., 118, 416-434, https://doi.org/10.1029/2012JD018658, 2013.

Holton, J. R.: The role of gravity wave induced drag and diffusion in the momentum budget of the mesosphere, J. Atmos. Sci., 39, 791-799, https://doi.org/10.1175/15200469(1982)039<0791:TROGWI>2.0.CO;2, 1982.

Jacobi, C., Fröhlich, K., and Pogoreltsev, A.: Quasi twoday-wave modulation of gravity wave flux and consequences for the planetary wave propagation in a simple circulation model, J. Atmos. Sol.-Terr. Phy., 68, 283-292, https://doi.org/10.1016/j.jastp.2005.01.017, 2006.

Jacobi, C., Fröhlich, K., Portnyagin, Y., Merzlyakov, E., Solovjova, T., Makarov, N., Rees, D., Fahrutdinova, A., Guryanov, V., Fedorov, D., Korotyshkin, D., Forbes, J., Pogoreltsev, A., and Kürschner, D.: Semi-empirical model of middle atmosphere wind from the ground to the lower thermosphere, Adv. Space Res., 43, 239-246, https://doi.org/10.1016/j.asr.2008.05.011, 2009.

Jacobi, C., Lilienthal, F., Geißler, C., and Krug, A.: Long-term variability of mid-latitude mesosphere-lower thermosphere winds over Collm $\left(51^{\circ} \mathrm{N}, 13^{\circ} \mathrm{E}\right)$, J. Atmos. Sol.-Terr. Phy., 136, 174186, https://doi.org/10.1016/j.jastp.2015.05.006, 2015.

Jakobs, H. J., Bischof, M., Ebel, A., and Speth, P.: Simulation of gravity wave effects under solstice conditions using a 3-D circulation model of the middle atmosphere, J. Atmos. Terr. Phys., 48, 1203-1223, https://doi.org/10.1016/0021-9169(86)90040-1, 1986.

Jiang, J. H., Wang, B., Goya, K., Hocke, K., Eckermann, S. D., Ma, J., Wu, D. L., and Read, W. J.: Geographical distribution and interseasonal variability of tropical deep convection: UARS
MLS observations and analyses, J. Geophys. Res., 109, D03111, https://doi.org/10.1029/2003JD003756, 2004.

Karami, K., Braesicke, P., Sinnhuber, M., and Versick, S.: On the climatological probability of the vertical propagation of stationary planetary waves, Atmos. Chem. Phys., 16, 8447-8460, https://doi.org/10.5194/acp-16-8447-2016, 2016.

Kirkwood, S., Mihalikova, M., Rao, T. N., and Satheesan, K.: Turbulence associated with mountain waves over Northern Scandinavia - a case study using the ESRAD VHF radar and the WRF mesoscale model, Atmos. Chem. Phys., 10, 3583-3599, https://doi.org/10.5194/acp-10-3583-2010, 2010.

Kumar, K., Ramkumar, T. K., and Krishnaiah, M.: Analysis of large-amplitude stratospheric mountain wave event observed from the AIRS and MLS sounders over the western Himalayan region, J. Geophys. Res., 117, D22102, https://doi.org/10.1029/2011JD017410, 2012.

Labitzke, K.: The Amplification of Height Wave 1 in January 1979: A Characteristic Precondition for the Major Warming in February, Mon. Weather Rev., 109, 983-989, https://doi.org/10.1175/15200493(1981)109<0983:TAOHWI>2.0.CO;2, 1981.

Li, Q., Graf, H.-F., and Giorgetta, M. A.: Stationary planetary wave propagation in Northern Hemisphere winter - climatological analysis of the refractive index, Atmos. Chem. Phys., 7, 183200, https://doi.org/10.5194/acp-7-183-2007, 2007.

Lieberman, R. S., Riggin, D. M., and Siskind, D. E.: Stationary waves in the wintertime mesosphere: Evidence for gravity wave filtering by stratospheric planetary waves, J. Geophys. Res., 118, 3139-3149, https://doi.org/10.1002/jgrd.50319, 2013.

Lilienthal, F., Jacobi, C., Schmidt, T., de la Torre, A., and Alexander, P.: On the influence of zonal gravity wave distributions on the Southern Hemisphere winter circulation, Ann. Geophys., 35, 785-798, https://doi.org/10.5194/angeo-35-785-2017, 2017.

Lilienthal, F., Jacobi, C., and Geißler, C.: Forcing mechanisms of the terdiurnal tide, Atmos. Chem. Phys., 18, 15725-15742, https://doi.org/10.5194/acp-18-15725-2018, 2018.

Lilly, D. K., Nicholls, J. M., Kennedy, P. J., Klemp, J. B., and Chervin, R. M.: Aircraft measurements of wave momentum flux over the Colorado Rocky mountains, Q. J. Roy. Meteor. Soc., 108, 625-641, https://doi.org/10.1002/qj.49710845709, 1982.

Lindzen, R. S.: Turbulence and stress owing to gravity wave and tidal breakdown, J. Geophys. Res., 86, 9707-9714, https://doi.org/10.1029/JC086iC10p09707, 1981.

Llamedo, P., de la Torre, A., Luna, P. A. D., Schmidt, T., and Wickert, J.: A gravity wave analysis near the Andes range from GPS radio occultation data and mesoscale numerical simulations: Two case studies, Adv. Space Res., 44, 494-500, https://doi.org/10.1016/j.asr.2009.04.023, 2009.

Matsuno, T.: A dynamical model of the stratospheric sudden warming, J. Atmos. Sci., 28, 1479-1494, https://doi.org/10.1175/15200469(1971)028<1479:ADMOTS>2.0.CO;2, 1971.

Matthias, V. and Ern, M.: On the origin of the mesospheric quasi-stationary planetary waves in the unusual Arctic winter 2015/2016, Atmos. Chem. Phys., 18, 4803-4815, https://doi.org/10.5194/acp-18-4803-2018, 2018.

Moffat-Griffin, T., Hibbins, R. E., Jarvis, M. J., and Colwell, S. R.: Seasonal variation of gravity wave activity in the lower strato- 
sphere over an Antarctic Peninsula station, J. Geophys. Res., 116, D14111, https://doi.org/10.1029/2010JD015349, 2010.

Nastrom, G. D. and Fritts, D. C.: Sources of Mesoscale Variability of Gravity Waves. Part I: Topographic Excitation, J. Atmos. Sci., 49, 101-110, https://doi.org/10.1175/15200469(1992)049<0101:SOMVOG>2.0.CO;2, 1992.

Pišoft, P., Šácha, P., Miksovsky, J., Huszar, P., Scherllin-Pirscher, B., and Foelsche, U.: Revisiting internal gravity waves analysis using GPS RO density profiles: comparison with temperature profiles and application for wave field stability study, Atmos. Meas. Tech., 11, 515-527, https://doi.org/10.5194/amt-11-5152018, 2018.

Plougonven, R. and Zhang, F.: Internal gravity waves from atmospheric jets and fronts, Rev. Geophys., 52, 33-76, https://doi.org/10.1002/2012RG000419, 2014.

Plougonven, R., Hertzog, A., and Teitelbaum, H.: Observations and simulations of a large-amplitude mountain wave breaking over the Antarctic Peninsula, J. Geophys. Res., 113, D16113, https://doi.org/10.1029/2007JD009739, 2008.

Pogoreltsev, A. I., Vlasov, A. A., Fröhlich, K., and Jacobi, C.: Planetary waves in coupling the lower and upper atmosphere, J. Atmos. Solar-Terr. Phys., 69, 2083-2101, https://doi.org/10.1016/j.jastp.2007.05.014, 2007.

Portnyagin, Y., Solovjova, T., Merzlyakov, E., Forbes, J., Palo, S., Ortland, D., Hocking, W., MacDougall, J., Thayaparan, T., Manson, A., Meek, C., Hoffmann, P., Singer, W., Mitchell, N., Pancheva, D., Igarashi, K., Murayama, Y., Jacobi, C., Kürschner, D., Fahrutdinova, A., Korotyshkin, D., Clark, R., Tailor, M., Franke, S., Fritts, D., Tsuda, T., Nakamura, T., Gurubaran, S., Rajaram, R., Vincent, R., Kovalam, S., Batista, P., Poole, G., Malinga, S., Fraser, G., Murphy, D., Riggin, D., Aso, T., and Tsutsumi, M.: Mesosphere/lower thermosphere prevailing wind model, Adv. Space Res., 34, 1755-1762, https://doi.org/10.1016/j.asr.2003.04.058, 2004.

Reid, I. M. and Vincent, R. A.: Measurements of mesospheric gravity wave momentum fluxes and mean flow accelerations at Adelaide, Australia, J. Atmos. Terr. Phys., 49, 443-460, https://doi.org/10.1016/0021-9169(87)90039-0, 1987.

Šácha, P., Kuchař, A., Jacobi, C., and Pišoft, P.: Enhanced internal gravity wave activity and breaking over the northeastern Pacificeastern Asian region, Atmos. Chem. Phys., 15, 13097-13112, https://doi.org/10.5194/acp-15-13097-2015, 2015.

Šácha, P., Lilienthal, F., Jacobi, C., and Pišoft, P.: Influence of the spatial distribution of gravity wave activity on the middle atmospheric dynamics, Atmos. Chem. Phys., 16, 15755-15775, https://doi.org/10.5194/acp-16-15755-2016, 2016.
Šácha, P., Miksovsky, J., and Pisoft, P.: Interannual variability in the gravity wave drag - vertical coupling and possible climate links, Earth Syst. Dynam., 9, 647-661, https://doi.org/10.5194/esd-9647-2018, 2018

Schmidt, T., Alexander, P., and de la Torre, A.: Stratospheric gravity wave momentum flux from radio occultations, J. Geophys. Res., 121, 4443-4467, https://doi.org/10.1002/2015JD024135, 2016.

Smith, A. K.: The origin of stationary planetary waves in the upper mesosphere, J. Atmos. Sci., 60, 3033-3041, https://doi.org/10.1175/15200469(2003)060<3033:TOOSPW>2.0.CO;2, 2003.

Smith, R. B.: On Severe Downslope Winds, J. Atmos. Sci., 42, 2597-2603, https://doi.org/10.1175/15200469(1985)042<2597:OSDW>2.0.CO;2, 1985.

Strobel, D. F.: Parameterization of the atmospheric heating rate from 15 to $120 \mathrm{~km}$ due to $\mathrm{O}_{2}$ and $\mathrm{O}_{3}$ Absorption of solar radiation, J. Geophys. Res., 83, 6225-6230, https://doi.org/10.1029/JC083iC12p06225, 1986.

Swinbank, R. and Ortland, D. A.: Compilation of wind data for the Upper Atmosphere Research Satellite (UARS) Reference Atmosphere Project, J. Geophys. Res., 108, 4615, https://doi.org/10.1029/2002JD003135, 2003.

Tsuda, T., Muayama, Y., Wiryosumarto, H., Harijono, S. W. B., and Kato, S.: Radiosonde observations of equatorial atmosphere dynamics over Indonesia. II: characteristics of gravity waves, J. Geophys. Res., 99, 10507-10516, https://doi.org/10.1029/94JD00354, 1994.

White, R. H., Battisti, D. S., and Sheshadri, A.: Orography and the Boreal Winter Stratosphere: The Importance of the Mongolian Mountains, Geophys. Res. Lett., 45, 2088-2096, https://doi.org/10.1002/2018GL077098, 2018.

Wright, C. J. and Gille, J. C.: HIRDLS observations of gravity wave momentum fluxes over the monsoon regions, J. Geophys. Res., 116, https://doi.org/10.1029/2011JD015725, 2011.

Xiao, C., Hu, X., and Tian, J.: Global temperature stationary planetary waves extending from 20 to $120 \mathrm{~km} \mathrm{ob-}$ served by TIMED/SABER, J. Geophys. Res., 114, D17101, https://doi.org/10.1029/2008JD011349, 2009. 